\title{
Gene expression profiling of sepsis-associated acute kidney injury
}

\author{
JING-JUAN YANG ${ }^{1}$, BIN-BIN WU $^{2}$, FEI HAN ${ }^{2}$, JIANG-HUA CHEN ${ }^{2}$ and YI YANG ${ }^{1,2}$ \\ ${ }^{1}$ Department of Nephrology, The Fourth Affiliated Hospital, College of Medicine, \\ Zhejiang University, Yiwu, Zhejiang 322000; ${ }^{2}$ Kidney Disease Center, The First Affiliated Hospital, \\ College of Medicine, Zhejiang University, Hangzhou, Zhejiang 310000, P.R. China
}

Received May 23, 2019; Accepted June 19, 2020

DOI: 10.3892/etm.2020.9161

\begin{abstract}
Sepsis accounts for more than $50 \%$ of all acute kidney injury (AKI) cases, and the combination of sepsis and AKI increases the risk of mortality from sepsis alone. However, to the best of our knowledge, the specific mechanism by which sepsis causes AKI has not yet been fully elucidated, and there is no targeted therapy for sepsis-associated AKI (SA-AKI). The present study investigated gene expression profiles using RNA sequencing (RNA-Seq) and bioinformatics analyses to assess the function of differentially expressed genes (DEGs) and the molecular mechanisms relevant to the prognosis of SA-AKI. From the bioinformatics analysis, 2,256 downregulated and 3,146 upregulated genes were identified (false discovery rate $<0.1$ and fold-change $>2$ ). Gene Ontology analysis revealed that the genes were enriched in cellular metabolic processes, cell death and apoptosis. The enriched transcription factors were v-rel reticuloendotheliosis viral oncogene homolog A and signaling transducer and activator of transcription 3. The enriched microRNAs (miRNAs or miRs) among the DEGs were miR-30e, miR-181a, miR-340, miR-466d and miR-4661. Furthermore, the enriched pathways included toll-like receptor signaling, nod-like receptor signaling and the Janus kinase/STAT signaling pathway. In conclusion, the present study identified certain prognosis-related genes, transcription factors, miRNAs and pathways by analyzing gene expression profiles of SA-AKI using RNA-Seq, which provides some basis for future experimental studies.
\end{abstract}

\section{Introduction}

Sepsis is a clinical syndrome characterized by organ dysfunction caused by a dysregulated immune response to infection (1). Acute kidney injury (AKI) is a serious clinical problem with high levels of mortality (2). Sepsis accounts

Correspondence to: Dr Yi Yang, Department of Nephrology, The Fourth Affiliated Hospital, College of Medicine, Zhejiang University, Yiwu, Zhejiang 322000, P.R. China

E-mail: yangyixk@zju.edu.cn

Key words: sepsis, acute kidney injury, RNA sequencing, bioinformatics, gene expression profiling for more than $50 \%$ of all AKI cases, and the combination of sepsis and AKI increases the risk of mortality compared with sepsis alone $(3,4)$. However, to the best of our knowledge, the specific mechanism by which sepsis causes AKI has not yet been fully elucidated, and there is no targeted therapy for sepsis-associated AKI (SA-AKI).

Although the pathophysiological mechanism has not yet been elucidated, it has been suggested that the deleterious inflammatory cascade characteristic of sepsis contributes to AKI (5). Compared with patients with sepsis without AKI, those with AKI have a higher risk of mortality $(6,7)$. However, the renal function of some patients with SA-AKI can return to a normal level (8). A previous study reported that resuscitation of lipopolysaccharide-treated mice with warmed $0.9 \%$ saline can increase survival rates to $>80 \%$, which allows to study the prognosis of SA-AKI (9). It is therefore vital to investigate the heterogeneity and molecular mechanisms related to the prognosis of SA-AKI, which may provide new therapeutic targets for SA-AKI.

RNA sequencing (RNA-Seq) has become a routine technique for genome-wide expression analysis as it can provide high-resolution sequence information (10). The present study investigated gene expression profiles using RNA-Seq and subsequent bioinformatics analyses to assess the function of differentially expressed genes (DEGs) and molecular mechanisms relevant to the prognosis of SA-AKI.

\section{Materials and methods}

Mouse cecal ligation and puncture (CLP) model. A total of 20 8-week-old male C57BL/6J mice (weight, 20-25 g) were obtained from Shanghai SLAC Laboratory Animal Co., Ltd., and housed in a room at $22 \pm 1^{\circ} \mathrm{C}, 40-60 \%$ humidity, with a $12-\mathrm{h}$ light/dark cycle (8:00 a.m.-8:00 p.m.), with free access to food and drinking water. All operations on mice were performed by one individual who was skilled in animal modeling. As described previously (9), a 1-cm midline laparotomy was performed following anesthesia with 3\% inhaled isoflurane. The base of the cecum was ligated and punctured at its distal tip using a single 21-gauge needle. Subsequently, a small amount of stool content (about $1 \mathrm{~mm}$ length) was extruded and put back into the abdominal cavity. The abdomen was sealed and $1 \mathrm{ml}$ warm sterile $0.9 \%$ saline was injected subcutaneously to resuscitate mice. The sham group $(n=8)$ underwent laparotomy and cecum exposure but no cecal ligation or puncture. 
A
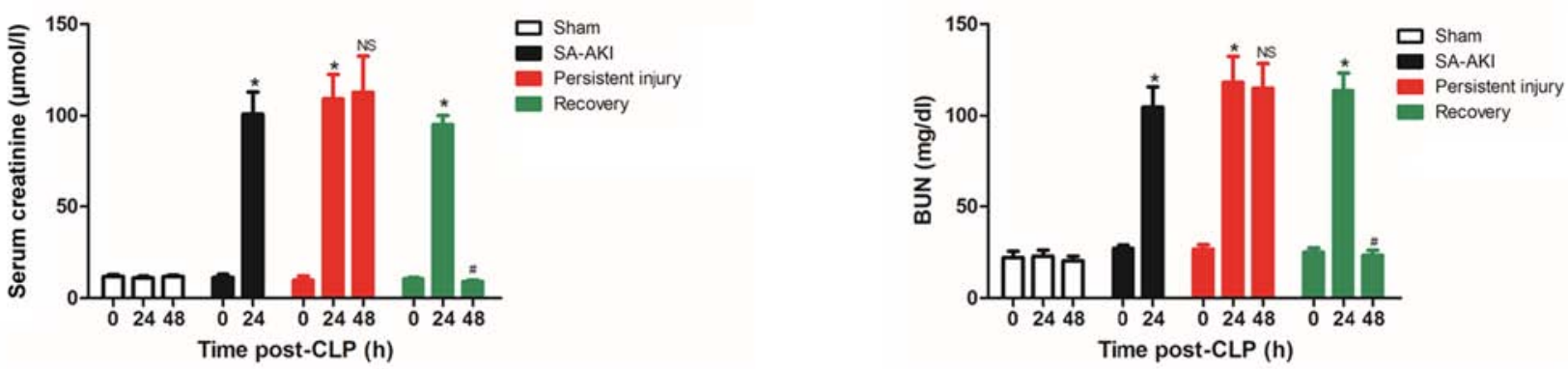

B

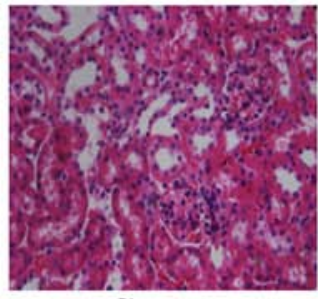

Sham

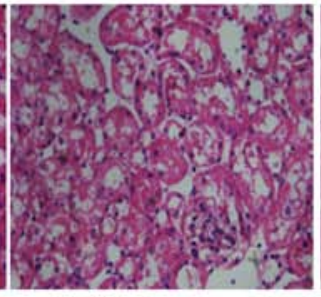

SA-AKI

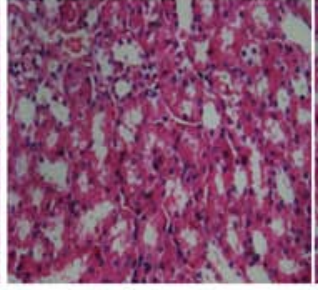

Recovery

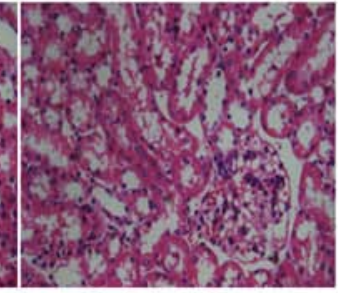

Persistent injury

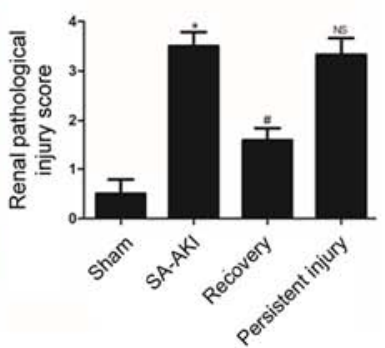

Figure 1. Renal function and renal structures in SA-AKI mice. (A) A total of $24 \mathrm{~h}$ post-CLP, mice received $10 \mathrm{ml} / \mathrm{kg}$ saline, and by $48 \mathrm{~h}$ post-CLP, a number of mice had recovered from AKI. (B) Hematoxylin-eosin staining revealed micro-vacuolization in proximal tubular cells in SA-AKI mice. The renal tissue pathological injury score in the SA-AKI group was significantly higher compared with that in the sham and recovery groups. There was no significant difference in the renal tissue pathological injury score between the SA-AKI and persistent injury groups. Magnification, $\mathrm{x} 400$. All experiments were performed a minimum of three times, and the results are presented as the mean \pm standard deviation. ${ }^{*} \mathrm{P}<0.05$ vs. Sham group. ${ }^{\#} \mathrm{P}<0.05$ vs. SA-AKI group. ${ }^{\mathrm{N}} \mathrm{P}<0.05$ vs. SA-AKI group. SA-AKI, sepsis-associated acute kidney injury; CLP, cecal ligation and puncture.

A
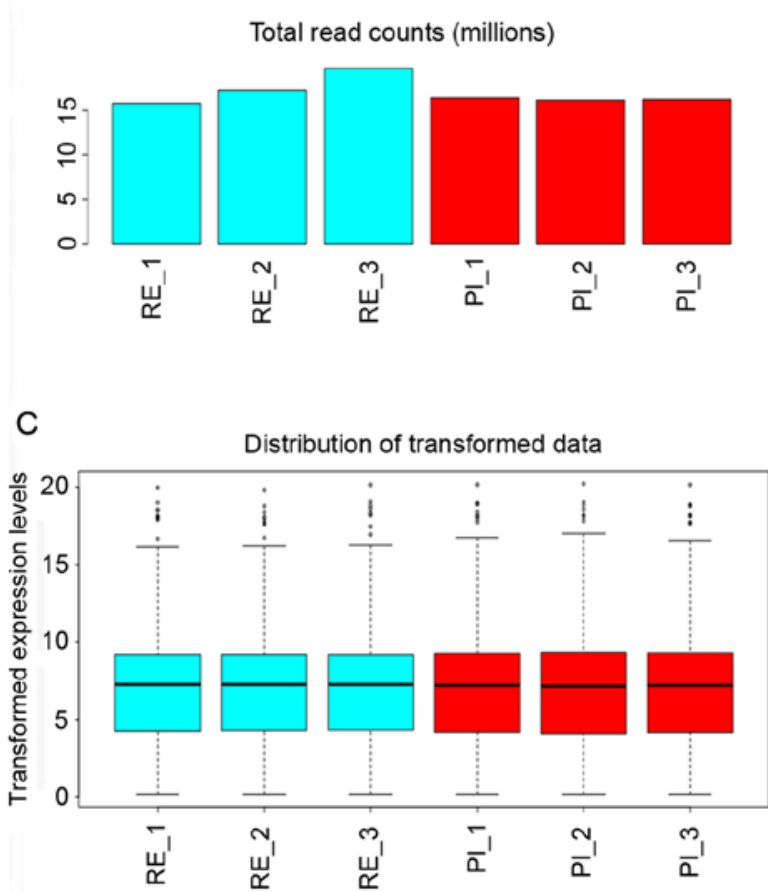

B

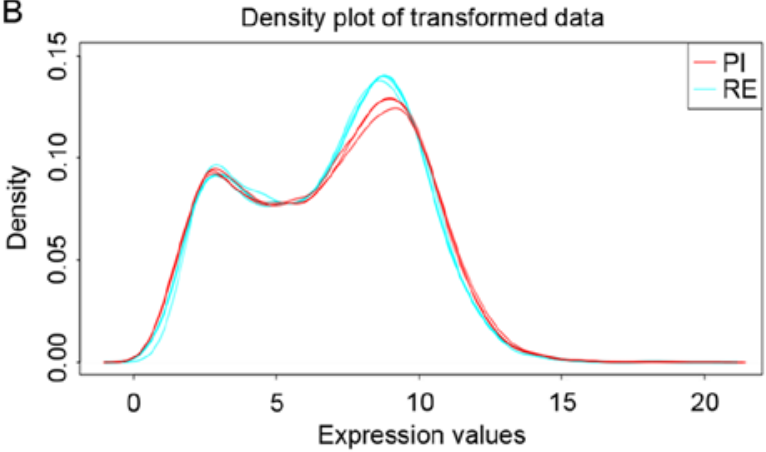

D

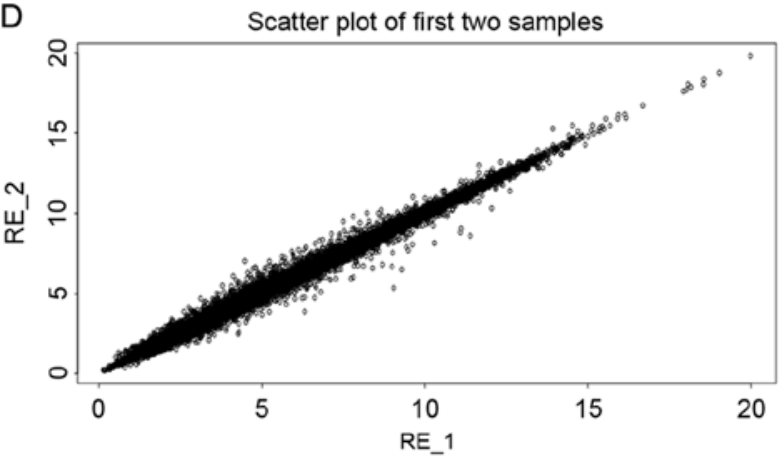

Figure 2. Diagnostic plots for read-counts data. (A) Total read-counts in the RE and PI samples. (B) Distribution of transformed data as presented using a density plot. (C) Boxplot of transformed data. (D) Scatter plot of the first two samples: RE_1 and RE_2. RE, recovery; PI, persistent injury.

At $24 \mathrm{~h}$ or $48 \mathrm{~h}$ post-surgery, all mice were anesthetized by inhalation of $3 \%$ isoflurane, and then $50 \mu 1$ retro-orbital venous blood was collected to measure Scr and BUN levels. Following collection of retro-orbital venous blood to assess
Scr and BUN levels, 4 mice were sacrificed ( $24 \mathrm{~h}$ post-CLP, SA-AKI group) and 8 mice were given an additional $10 \mathrm{ml} / \mathrm{kg}$ warmed sterile $0.9 \%$ saline, and then sacrificed at $48 \mathrm{~h}$ post-CLP by cervical dislocation. AKI was defined 


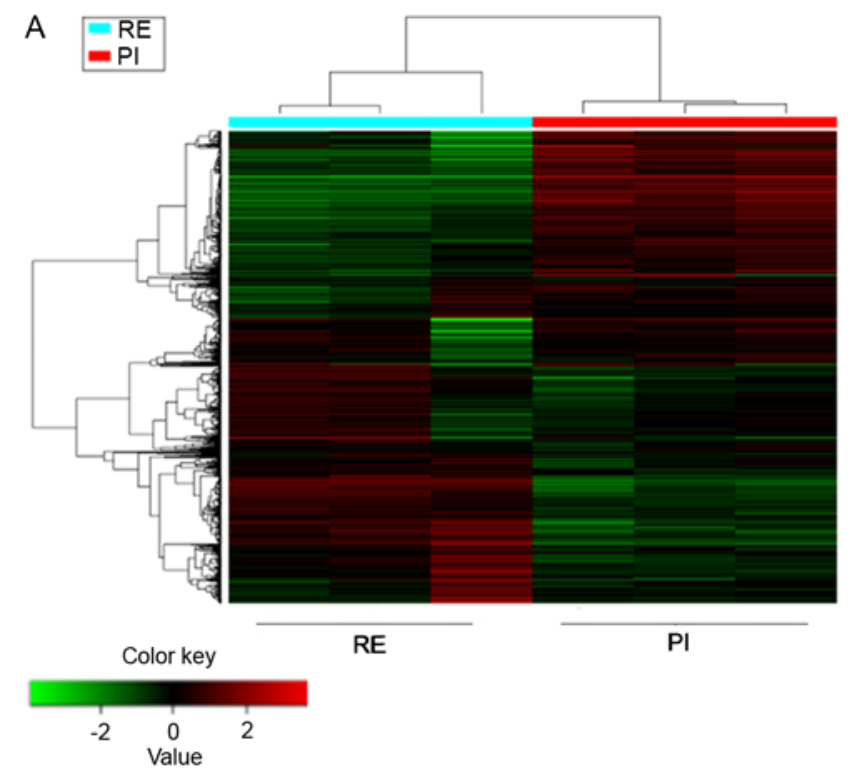

B

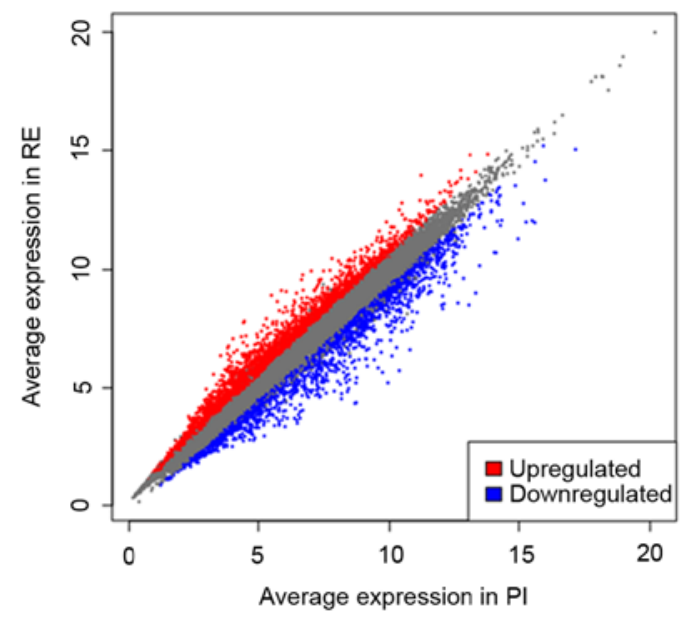

C

D
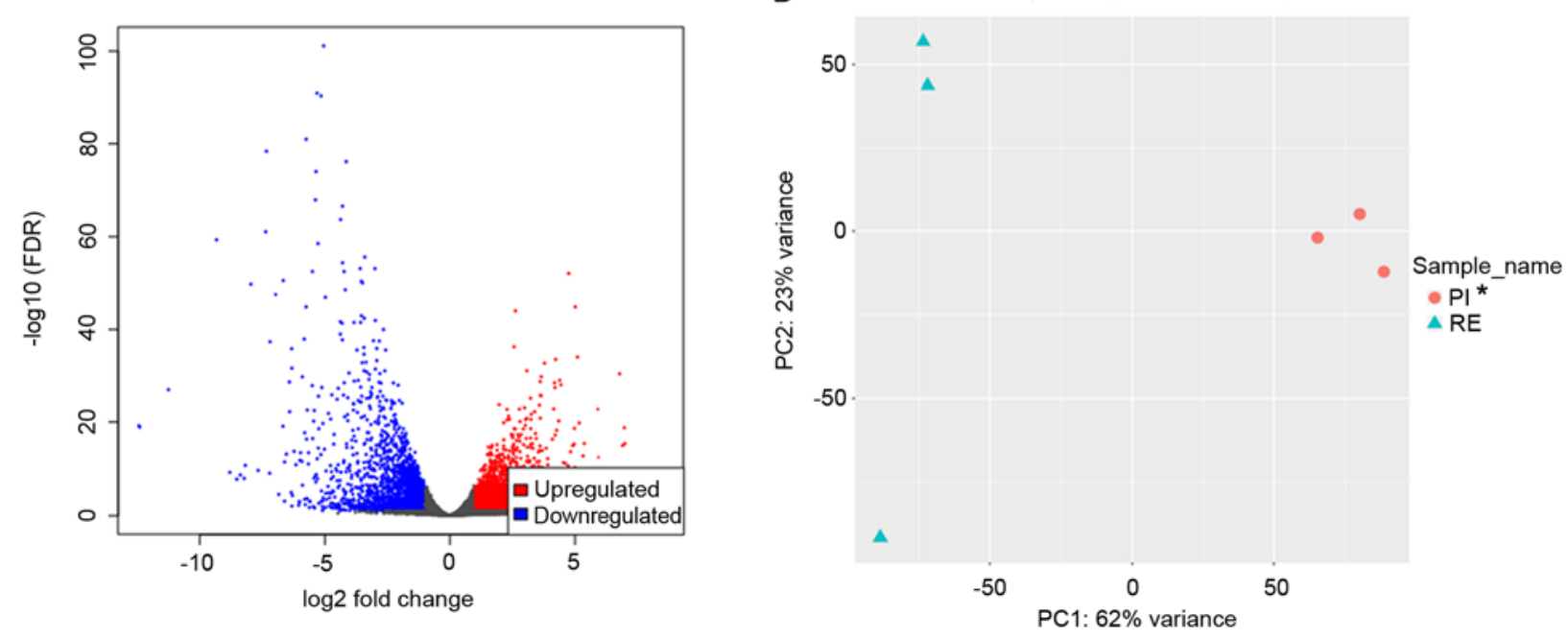

Figure 3. Bioinformatics analysis of mRNA expression patterns in the PI and RE groups by RNA-Seq. (A) Hierarchical clustering was used to evaluate the top 2,000 genes with significant differential expression in the PI and RE group samples. Each column represents one samples expression pattern. The red lines represent the upregulated genes, and the green lines represent the downregulated genes. (B) Scatter plot analysis was conducted to demonstrate the mRNA expression distribution. (C) The volcano plot was generated to visualize the significant differentially expressed genes. (D) Principle component analysis demonstrated that there was a significant difference between the RE and PI group samples, along the first principal component that explained $62 \%$ of the variance. ${ }^{~} \mathrm{P}<0.05$ vs. RE group; RE, recovery; PI, persistent injury; FDR, false discovery rate.

as a serum creatinine (Scr) level $>20 \mu \mathrm{mol} / 1$ and blood urea nitrogen $(\mathrm{BUN})>28.3 \mathrm{mg} / \mathrm{dl}$. Mice were grouped into persistent injury (PI; $n=3)$ and recovery $(\mathrm{RE} ; \mathrm{n}=5)$ groups according to renal function at $48 \mathrm{~h}$ post-CLP. The RE group consisted of mice whose Scr and BUN levels returned to normal $(<20 \mu \mathrm{mol} / 1$ and $<28.3 \mathrm{mg} / \mathrm{dl}$, respectively) at $48 \mathrm{~h}$. The PI group contained mice with high levels of $\mathrm{Scr}$ $(>20 \mu \mathrm{mol} / \mathrm{l})$ and BUN $(>28.3 \mathrm{mg} / \mathrm{dl})$ at $48 \mathrm{~h}$. Mice in the SA-AKI group were sacrificed to collect blood and kidneys at $24 \mathrm{~h}(\mathrm{n}=4)$. Mice in the PI $(\mathrm{n}=3)$ and RE $(\mathrm{n}=5)$ group were sacrificed to collect blood and kidneys at $48 \mathrm{~h}$. Mice in the sham group were sacrificed via cervical dislocation to collect blood and kidneys at $24 \mathrm{~h}(\mathrm{n}=4)$ and $48 \mathrm{~h}(\mathrm{n}=4)$, respectively.

All protocols of this study were approved by the Animal Ethics Committee of the First Affiliated Hospital, College of Medicine, Zhejiang University, Hangzhou, Zhejiang (approval
ID. 2016160; 25 February 2016), which follows the institutional guidelines.

Renal function assessment. Blood samples were centrifuged at $13,000 \mathrm{x}$ g for $10 \mathrm{~min}$ at $4^{\circ} \mathrm{C}$ to collect the supernatant. Scr and BUN levels were measured using the DRI-CHEM dry biochemical analyzer (FUJIFILM Wako Pure Chemical Corporation).

Hematoxylin-eosin staining. The kidney tissues of mice were fixed in $4 \%$ paraformaldehyde at room temperature overnight, embedded in paraffin wax, sliced into 4-mm-thick sections, and stained with hematoxylin and eosin at $37^{\circ} \mathrm{C}$ for $24 \mathrm{~h}$. Slides were prepared in triplicate, and sections were evaluated under a light microscope (magnification, $\mathrm{x} 400$ ). The pathological injury score of all the samples was assessed twice in a blinded fashion by independent investigators, and the mean score from 
Table I. Enrichment analysis of k-means clusters using various gene sets.

\begin{tabular}{|c|c|c|c|}
\hline Cluster & Adjusted P-value & Genes (n) & Pathway \\
\hline A & $2.03 \times 10^{-5}$ & 42 & GO:0009605 response to external stimulus \\
\hline A & 0.000133 & 40 & GO:0048468 cell development \\
\hline A & 0.000311 & 17 & GO:0006935 chemotaxis \\
\hline A & 0.000311 & 31 & GO:0040011 locomotion \\
\hline A & 0.000555 & 32 & GO:0022610 biological adhesion \\
\hline A & 0.000555 & 8 & GO:0098754 detoxification \\
\hline A & 0.000613 & 4 & GO:0015671 oxygen transport \\
\hline A & 0.000613 & 27 & GO:0032989 cellular component morphogenesis \\
\hline A & 0.0009 & 31 & GO:0007155 cell adhesion \\
\hline A & 0.001458 & 31 & GO:0006928 movement of cell or subcellular component \\
\hline A & 0.001516 & 25 & GO:0016477 cell migration \\
\hline A & 0.001626 & 24 & GO:0048646 anatomical structure formation involved in morphogenesis \\
\hline A & 0.001668 & 19 & GO:0040012 regulation of locomotion \\
\hline B & 0.000751 & 46 & GO:0006082 organic acid metabolic process \\
\hline $\mathrm{B}$ & 0.000751 & 26 & GO:0006631 fatty acid metabolic process \\
\hline $\mathrm{B}$ & 0.000751 & 33 & GO:0032787 monocarboxylic acid metabolic process \\
\hline $\mathrm{B}$ & 0.000991 & 41 & GO:0019752 carboxylic acid metabolic process \\
\hline B & 0.001931 & 7 & GO:0060740 prostate gland epithelium morphogenesis \\
\hline $\mathrm{B}$ & 0.004579 & 8 & GO:0030850 prostate gland development \\
\hline $\mathrm{B}$ & 0.004829 & 6 & GO:0055081 anion homeostasis \\
\hline $\mathrm{B}$ & 0.00809 & 52 & GO:0006629 lipid metabolic process \\
\hline $\mathrm{B}$ & 0.00809 & 5 & GO:0060572 morphogenesis of an epithelial bud \\
\hline $\mathrm{C}$ & $2.48 \times 10^{-46}$ & 109 & GO:0034097 response to cytokine \\
\hline $\mathrm{C}$ & $5.84 \times 10^{-42}$ & 174 & GO:0009605 response to external stimulus \\
\hline $\mathrm{C}$ & $2.22 \times 10^{-41}$ & 135 & GO:0006952 defense response \\
\hline $\mathrm{C}$ & $1.04 \times 10^{-40}$ & 174 & GO:0002376 immune system process \\
\hline $\mathrm{C}$ & $1.71 \times 10^{-38}$ & 168 & GO:0071310 cellular response to organic substance \\
\hline $\mathrm{C}$ & $2.96 \times 10^{-36}$ & 104 & GO:0009607 response to biotic stimulus \\
\hline $\mathrm{C}$ & $2.96 \times 10^{-36}$ & 101 & GO:0043207 response to external biotic stimulus \\
\hline $\mathrm{C}$ & $2.96 \times 10^{-36}$ & 88 & GO:0071345 cellular response to cytokine stimulus \\
\hline $\mathrm{C}$ & $3.22 \times 10^{-34}$ & 153 & GO:0008219 cell death \\
\hline $\mathrm{C}$ & $3.57 \times 10^{-34}$ & 118 & GO:0006955 immune response \\
\hline $\mathrm{C}$ & $6.62 \times 10^{-34}$ & 64 & GO:0032496 response to lipopolysaccharide \\
\hline $\mathrm{C}$ & $7.23 \times 10^{-34}$ & 147 & GO:0012501 programmed cell death \\
\hline $\mathrm{C}$ & $1.98 \times 10^{-33}$ & 145 & GO:0006915 apoptotic process \\
\hline $\mathrm{C}$ & $3.00 \times 10^{-33}$ & 148 & GO:0048584 positive regulation of response to stimulus \\
\hline $\mathrm{D}$ & $6.27 \times 10^{-8}$ & 25 & GO:0034097 response to cytokine \\
\hline $\mathrm{D}$ & $1.24 \times 10^{-8}$ & 8 & GO:0035458 cellular response to interferon-beta \\
\hline $\mathrm{D}$ & $4.1 \times 10^{-6}$ & 7 & GO:0006953 acute-phase response \\
\hline $\mathrm{D}$ & $5.58 \times 10^{-5}$ & 27 & GO:0006952 defense response \\
\hline $\mathrm{D}$ & $5.77 \times 10^{-5}$ & 18 & GO:0071345 cellular response to cytokine stimulus \\
\hline $\mathrm{D}$ & $7.70 \times 10^{-5}$ & 35 & GO:0009605 response to external stimulus \\
\hline $\mathrm{D}$ & 0.00017 & 8 & GO:0034341 response to interferon-gamma \\
\hline $\mathrm{D}$ & 0.000215 & 14 & GO:0051346 negative regulation of hydrolase activity \\
\hline $\mathrm{D}$ & 0.000269 & 6 & GO:0050994 regulation of lipid catabolic process \\
\hline $\mathrm{D}$ & 0.000509 & 13 & GO:0052548 regulation of endopeptidase activity \\
\hline $\mathrm{D}$ & 0.000515 & 27 & GO:1901700 response to oxygen-containing compound \\
\hline $\mathrm{D}$ & 0.00062 & 3 & GO:0010727 negative regulation of hydrogen peroxide metabolic process \\
\hline $\mathrm{D}$ & 0.00062 & 4 & GO:0035634 response to stilbenoid \\
\hline
\end{tabular}




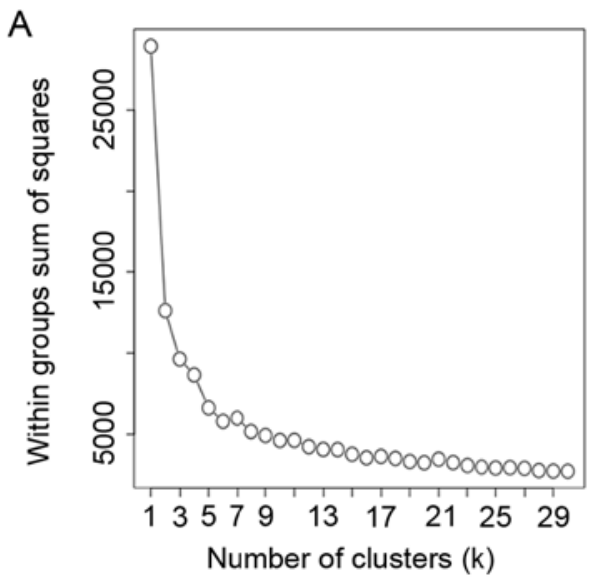

B

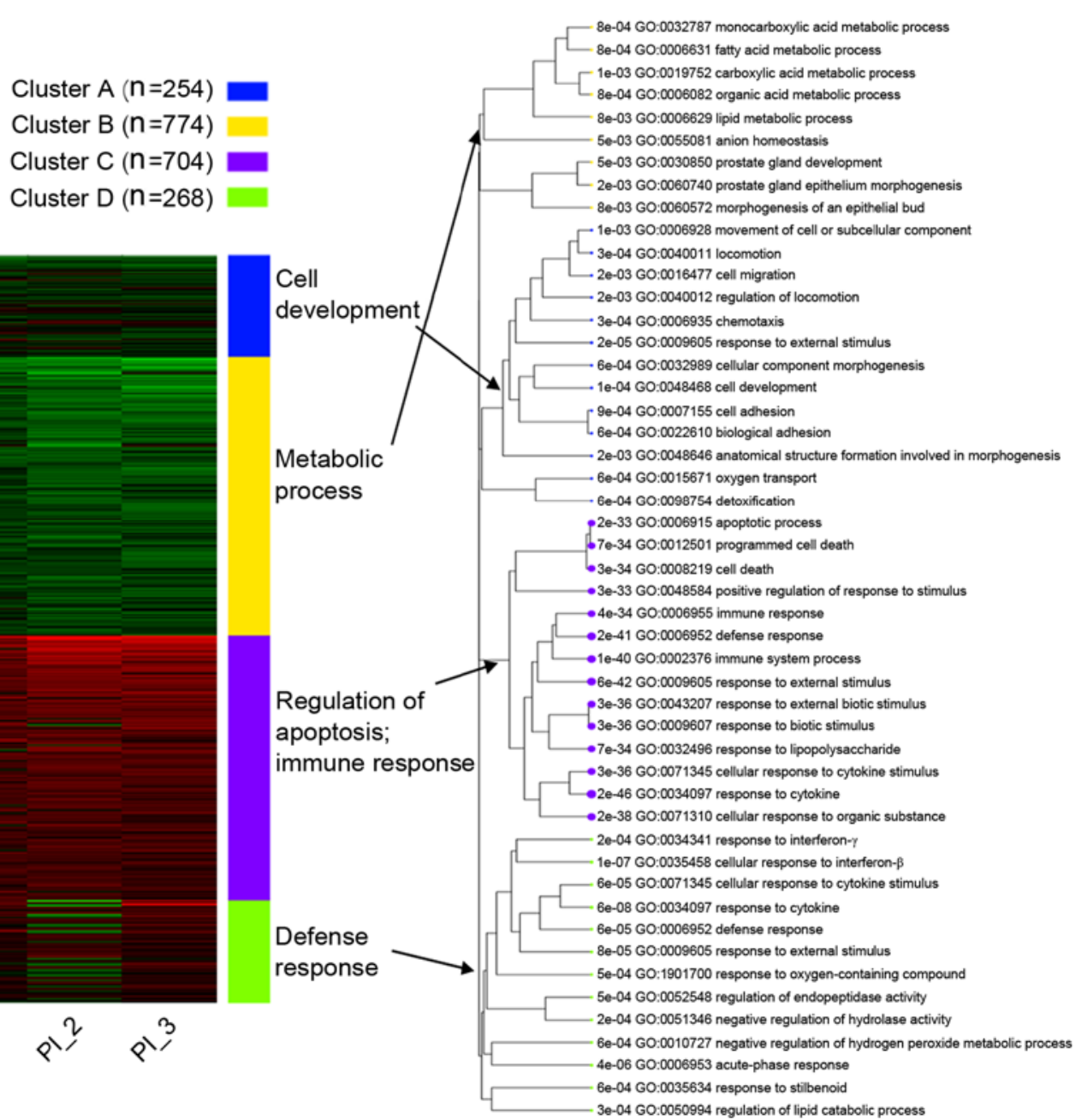

Figure 4. $\kappa$-means clustering and GO enrichment analysis of the RE and PI group samples. (A) Based on the within-group sum of squares plot as a reference, a larger $\kappa=4$ was selected. (B) The clusters A, B, C and D were predominantly enriched in cell development, metabolic process, regulation of apoptosis and immune response, and defense response, respectively. RE, recovery; PI, persistent injury; GO, Gene Ontology.

the two assessments was recorded as the composite score. The grading criteria for renal injury were as follows: Edema of tubular epithelial cells, loss of normal morphology, and cytoplasmic vacuoles. The grading standard for renal tubular involvement were as follows: Observing the aforementioned characteristics of the samples under the same microscope at x400 magnification. Renal tissue pathological injury score was assessed with a 0-5 scoring system: 0 , no renal tubular involvement; $1,<25 \%$ renal tubular involvement; $2,25-<50 \%$ renal tubular involvement; $3,50-75 \%$ renal tubular involvement; 4, >75\% renal tubular involvement.

RNA-Seq and gene expression analysis. Total RNA of 3 kidneys in the PI group and 3 kidneys in the RE group were extracted using TRlzol ${ }^{\circledR}$ reagent (Invitrogen; Thermo Fisher Scientific, Inc.) according to the manufacturer's instructions. RNA purity 
Table II. Enriched GO Biological Process terms for the upregulated and downregulated genes.

A, Downregulated genes

\begin{tabular}{lll}
\hline Adjusted P-value & Genes $(\mathrm{n})$ & \multicolumn{1}{c}{ Biological process } \\
\hline$<0.001$ & 362 & GO:0071310 cellular response to organic substance \\
$<0.001$ & 185 & GO:0034097 response to cytokine \\
$<0.001$ & 358 & GO:0002376 immune system process \\
$<0.001$ & 343 & GO:0009605 response to external stimulus \\
$<0.001$ & 315 & GO:0048584 positive regulation of response to stimulus \\
$<0.001$ & 321 & GO:0008219 cell death \\
$<0.001$ & 155 & GO:0071345 cellular response to cytokine stimulus \\
$<0.001$ & 243 & GO:0006952 defense response \\
$<0.001$ & 141 & GO:0001817 regulation of cytokine production \\
$<0.001$ & 298 & GO:0006915 apoptotic process \\
$<0.001$ & 301 & GO:0012501 programmed cell death \\
$<0.001$ & 180 & GO:0051707 response to other organism \\
$<0.001$ & 149 & GO:0001816 cytokine production \\
$<0.001$ & 214 & GO:0080134 regulation of response to stress \\
\hline
\end{tabular}

B, Upregulated genes

\begin{tabular}{lcl}
\hline Adjusted P-value & Genes $(\mathrm{n})$ & \multicolumn{1}{c}{ Biological process } \\
\hline$<0.001$ & 293 & GO:0044281 small molecule metabolic process \\
$<0.001$ & 151 & GO:0006082 organic acid metabolic process \\
$<0.001$ & 138 & GO:0019752 carboxylic acid metabolic process \\
$<0.001$ & 139 & GO:0043436 oxoacid metabolic process \\
$<0.001$ & 186 & GO:0044711 single-organism biosynthetic process \\
$<0.001$ & 99 & GO:0032787 monocarboxylic acid metabolic process \\
$<0.001$ & 61 & GO:0060271 cilium morphogenesis \\
$<0.001$ & 147 & GO:0044255 cellular lipid metabolic process \\
$<0.001$ & 183 & GO:0006629 lipid metabolic process \\
$<0.001$ & 72 & GO:0006631 fatty acid metabolic process \\
$<0.001$ & 40 & GO:0044782 cilium organization \\
$<0.001$ & 150 & GO:0019637 organophosphate metabolic process \\
$<0.001$ & 163 & GO:0055114 oxidation-reduction process \\
$<0.001$ & 76 & GO:0006820 anion transport \\
$<0.001$ & 54 & GO:0044282 small molecule catabolic process \\
\hline
\end{tabular}

GO, Gene Ontology.

was assessed using the kaiaoK5500 ${ }^{\circledR}$ Spectrophotometer (Kaiao). RNA integrity and concentration were detected using the RNA Nano 6000 assay kit of the Bioanalyzer 2100 system (Agilent Technologies, Inc.). A total amount of $2 \mu \mathrm{g}$ RNA per sample was used as input material for the RNA sample preparations. Sequencing libraries were built using the NEBNext $^{\circledR}$ Ultra $^{\text {TM }}$ RNA Library Prep kit for Illumina ${ }^{\circledR}$ (cat. no. E7530L; New England Biolabs, Inc.) following the manufacturer's instructions, and index codes were added to attribute sequences to each sample.

Based on the manufacturer's instructions, the index-coded samples were clustered on a cBot cluster generation system using HiSeq PE Cluster kit v4-cBot-HS (Illumina, Inc.). After the cluster was generated, the libraries were sorted on an Illumina platform to generate 150 bp paired-end reads. Genes with a false discovery rate (FDR) $<0.1$ and fold-change (FC) $>2$ were identified as DEGs.

Data analysis and pathway enrichment analysis were performed using integrated Differential Expression and Pathway analysis (iDEP, version 0.90; http://ge-lab. org/idep) (11). iDEP is an online application that integrates many Bioconductor packages and annotation databases to enable users to perform intensive bioinformatics analysis. In the present study, DESeq2 package, hierarchical clustering and $\kappa$-means clustering analysis, principal component analysis, Gene Ontology (GO) analysis, and transcription factor binding 
Table III. Enriched GO Cellular Component terms for the upregulated and downregulated genes.

A, Downregulated genes

\begin{tabular}{lrl}
\hline Adjusted P-value & Genes $(\mathrm{n})$ & \multicolumn{1}{c}{ Cellular component } \\
\hline$<0.001$ & 159 & GO:0005730 nucleolus \\
$<0.001$ & 115 & GO:0005912 adherens junction \\
$<0.001$ & 116 & GO:0070161 anchoring junction \\
$<0.001$ & 71 & GO:0005925 focal adhesion \\
$<0.001$ & 72 & GO:0030055 cell-substrate junction \\
$<0.001$ & 24 & GO:0030684 preribosome \\
$<0.001$ & 180 & GO:0030054 cell junction \\
$<0.001$ & 59 & GO:0005913 cell-cell adherens junction \\
$<0.001$ & 98 & GO:0005911 cell-cell junction \\
$<0.001$ & 108 & GO:0009986 cell surface \\
$<0.001$ & 36 & GO:0044452 nucleolar part \\
$<0.001$ & 13 & GO:0032040 small-subunit processome \\
$<0.001$ & 69 & GO:0098552 side of membrane \\
$<0.001$ & 178 & GO:0005615 extracellular space \\
\hline
\end{tabular}

B, Upregulated genes

\begin{tabular}{lcl}
\hline Adjusted P-value & Genes (n) & \multicolumn{1}{c}{ Cellular component } \\
\hline$<0.001$ & 104 & GO:0005929 cilium \\
$<0.001$ & 276 & GO:0042995 cell projection \\
$<0.001$ & 72 & GO:0044441 ciliary part \\
$<0.001$ & 35 & GO:0036064 ciliary basal body \\
$<0.001$ & 157 & GO:0044463 cell projection part \\
$<0.001$ & 176 & GO:0015630 microtubule cytoskeleton \\
$<0.001$ & 116 & GO:0005815 microtubule organizing center \\
$<0.001$ & 89 & GO:0005813 centrosome \\
$<0.001$ & 275 & GO:0005856 cytoskeleton \\
$<0.001$ & 46 & GO:0016323 basolateral plasma membrane \\
$<0.001$ & 243 & GO:0005739 mitochondrion \\
$<0.001$ & 141 & GO:0098590 plasma membrane region \\
$<0.001$ & 28 & GO:0005814 centriole \\
$<0.001$ & 13 & GO:0030990 intraciliary transport particle \\
$<0.001$ & 34 & GO:0044450 microtubule organizing center part \\
\hline
\end{tabular}

GO, Gene Ontology.

motifs and microRNA enrichment analysis were used in iDEP application.

Gene set enrichment analysis (GSEA). GSEA was conducted using the software GSEA v2.2.2 (www.broadinstitute.org/gsea). The enrichment degree and statistical significance were quantified by normalized enrichment score (NES), nominal P-value and FDR (12). Kyoto Encyclopedia of Genes and Genomes (KEGG) pathways were conducted by using GSEA software.

Statistical analysis. All data are presented as mean \pm standard deviation. The results were analyzed by one-way analysis of variance and Tukey's post hoc test using SPSS 22.0 software
(IBM Corp.). $\mathrm{P}<0.05$ was considered to indicate a statistically significant difference.

\section{Results}

Sepsis-associated AKImice.CLP was performed in 8-week-old male C57BL/6J mice. At $24 \mathrm{~h}$ after the CLP procedure, kidney function biomarkers BUN and Scr were significantly elevated. Following resuscitation, 5 mice exhibited recovery to normal kidney function by $48 \mathrm{~h}$ post-CLP operation, however 3 mice had persistent AKI. The levels of kidney function biomarkers are presented in Fig. 1A. It was identified that Scr and BUN levels did not significantly change in sham group at each time 

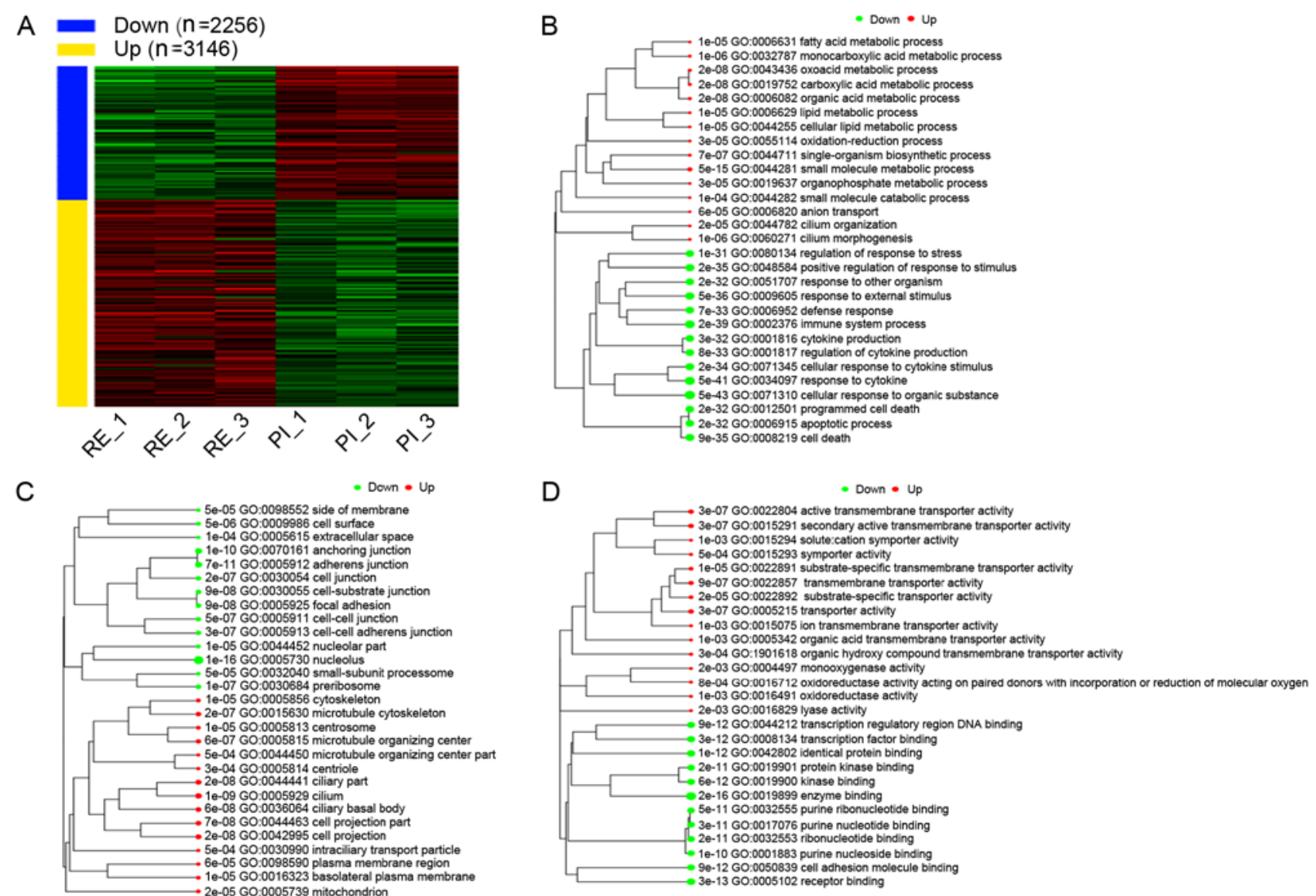

Figure 5. DEGs and enrichment analysis. (A) A total of 2,256 downregulated and 3,146 upregulated DEGs were identified in the RE group compared with the PI group using a threshold of false discovery rate $<0.1$ and fold-change $>2$. (B) The significantly enriched GO Biological Process terms for the upregulated and downregulated DEGs. (C) The significantly enriched GO Cellular Component terms for the upregulated and downregulated DEGs. (D) The significantly enriched GO Molecular Function terms for the upregulated and downregulated DEGs. DEGs, differentially expressed genes; GO, Gene Ontology; RE, recovery; PI, persistent injury.

point. Renal tissue pathological staining and injury score were used to evaluate renal injury in the present study. In the sham group, the kidney tissue was demonstrated to be normal and no signs of damage were present. To further quantify the degree of renal damage in each group of mice, the pathological grading of renal pathological sections was evaluated. The renal tissue pathological injury score in the SA-AKI group $(3.50 \pm 0.58)$ was significantly higher compared with that in the sham group $(0.50 \pm 0.58)$ and RE group $(1.60 \pm 0.55$; $\mathrm{P}<0.05)$. There was no significant difference in renal tissue pathological injury score between the SA-AKI and PI groups ( $3.50 \pm 0.58$ vs. $3.33 \pm 0.58 ; \mathrm{P}=0.72$; Fig. $1 \mathrm{~B})$. To investigate this heterogeneity, expression profiling was performed with the kidney-derived RNA obtained from the PI and RE groups $48 \mathrm{~h}$ post-CLP.

Expression profiling analyses of kidneys from mice with $S A-A K I$. iDEP was used to analyze the RNA-Seq dataset. The total read counts per library exhibited a small amount of variation in size (Fig. 2A). Regularized log transformation was performed using the DESeq2 package, and the distribution of the transformed data is presented in Fig. 2B and C, D presented the variation between the RE 1 and RE 2 samples to represent the variation among each replicate. Variation among each replicate was small.
Hierarchical clustering and $\mathrm{\kappa}-\mathrm{means}$ clustering analysis. Hierarchical clustering analysis evaluated the top 2,000 significant DEGs among all DEGs, including the 2256 downregulated and 3146 upregulated DEGs, which were ranked by their standard deviation across all samples. The results of hierarchical clustering analysis results are presented in Fig. 3A, in which each column represents the expression pattern of one sample, the red lines represent the upregulated genes, and the green lines represent the downregulated genes (Fig. 3A). A scatter plot was generated to depict the gene expression distribution (Fig. 3B), and a volcano map was created to visualize significant DEGs (Fig. 3C). A principal component analysis (PCA) plot using the first and second principal components is presented in Fig. 3D. There was a significant difference between the RE and PI groups along the first principal component, which explained the $62 \%$ variance.

Subsequently, $\kappa$-means clustering was used to divide the top 2,000 DEGs into groups. Based on the within-group sum of squares plot as a reference, $\kappa=4$ was selected and indicated the number of clusters used for further analysis (Fig. 4A). The four gene clusters underwent $\mathrm{GO}$ analysis and the results are presented in Fig. 4B and Table I.

Identification of DEGs. Compared with the PI group, a total of 2,256 downregulated and 3,146 upregulated DEGs were 
Table IV. Enriched GO Molecular Function terms for the upregulated and downregulated genes.

A, Downregulated genes

\begin{tabular}{lll}
\hline Adjusted P-value & Genes $(\mathrm{n})$ & \multicolumn{1}{c}{ Molecular function } \\
\hline$<0.001$ & 266 & GO:0019899 enzyme binding \\
$<0.001$ & 213 & GO:0005102 receptor binding \\
$<0.001$ & 204 & GO:0042802 identical protein binding \\
$<0.001$ & 100 & GO:0008134 transcription factor binding \\
$<0.001$ & 109 & GO:0019900 kinase binding \\
$<0.001$ & 133 & GO:0044212 transcription regulatory region DNA binding \\
$<0.001$ & 87 & GO:0050839 cell adhesion molecule binding \\
$<0.001$ & 256 & GO:0032553 ribonucleotide binding \\
$<0.001$ & 99 & GO:0019901 protein kinase binding \\
$<0.001$ & 254 & GO:0017076 purine nucleotide binding \\
$<0.001$ & 252 & GO:0032555 purine ribonucleotide binding \\
$<0.001$ & 247 & GO:0001883 purine nucleoside binding \\
\hline
\end{tabular}

B, Upregulated genes

\begin{tabular}{lrl}
\hline Adjusted P-value & Genes $(\mathrm{n})$ & \\
\hline$<0.001$ & 204 & GO:0005215 transporter activity \\
$<0.001$ & 56 & GO:0015291 secondary active transmembrane transporter activity \\
$<0.001$ & 78 & GO:0022804 active transmembrane transporter activity \\
$<0.001$ & 165 & GO:0022857 transmembrane transporter activity \\
$<0.001$ & 145 & GO:0022891 substrate-specific transmembrane transporter activity \\
$<0.001$ & 166 & GO:0022892 substrate-specific transporter activity \\
$<0.001$ & 13 & GO:1901618 organic hydroxy compound transmembrane transporter activity \\
$<0.001$ & 33 & GO:0015293 symporter activity \\
$<0.001$ & 18 & GO:0016712 oxidoreductase activity acting on paired donors with \\
& & incorporation or reduction of molecular oxygen reduced flavin or \\
$<0.001$ & & flavoprotein as one donor and incorporation of one atom of oxygen \\
0.001 & 124 & GO:0016491 oxidoreductase activity \\
0.001 & 27 & GO:0005342 organic acid transmembrane transporter activity \\
0.001 & 118 & GO:0015075 ion transmembrane transporter activity \\
0.002 & 24 & GO:0015294 solute:cation symporter activity \\
0.002 & 33 & GO:0004497 monooxygenase activity \\
\hline
\end{tabular}

GO, Gene Ontology.

identified in the RE group using a threshold of FDR $<0.1$ and FC $>2$ (Fig. 5A). The upregulated and downregulated DEGs then underwent GO analysis for Biological Process terms. In Fig. 5B, the upregulated DEGs were mainly involved in 'metabolic processes', the downregulated DEGs were enriched in 'regulation of response to stress', 'cytokine production', 'apoptotic process' and 'cell death'. The details of the enriched GO Biological Process terms are presented in Table II. For GO Cellular Component terms, it was identified that the upregulated DEGs in the RE group were enriched in the 'mitochondrion', 'cytoskeleton' and 'centrosome'; the downregulated DEGs in the RE group were enriched in the 'cell surface', 'cell junction' and 'adherens junction' (Fig. 5C and Table III). For GO
Molecular Function terms, it was identified the downregulated DEGs in the RE group were enriched in 'enzyme binding' and 'receptor binding'. Furthermore, the upregulated DEGs were enriched in 'transporter activity and active transmembrane transporter activity' (Fig. 5D and Table IV).

According to the enriched genes in the upregulated genes of the RE group, it was identified that solute carrier family 7 member 7 (Slc7a7) was highly enriched in the top six pathways (Table SI).

Transcription factor (TF) binding motifs and microRNA (miRNA or miR) target gene sets. As presented in Table V, using the TF target gene sets in the enrichment analysis, it was 
Table V. Enriched transcription factor binding motifs for the downregulated genes.

\begin{tabular}{lcl}
\hline Adjusted P-value & Genes $(\mathrm{n})$ & TF binding motifs \\
\hline$<0.001$ & 14 & TRANSFAC: RELA \\
$<0.001$ & 12 & TRANSFAC: STAT3 \\
$<0.001$ & 7 & TRANSFAC: $:$ REL \\
$<0.001$ & 14 & TRANSFAC: CEBPB \\
$<0.001$ & 12 & TRANSFAC: CREB1 \\
$<0.001$ & 10 & TRANSFAC: JUN \\
$<0.001$ & 8 & TRANSFAC: FOS \\
$<0.001$ & 11 & TRANSFAC: SFPI1 \\
$<0.001$ & 6 & TRANSFAC: CEBPD \\
0.002 & 3 & TRANSFAC: NFKB1 \\
\hline
\end{tabular}

detected that target genes of v-rel reticuloendotheliosis viral oncogene homolog A (RELA; FDR $\left.<1.92 \times 10^{-8}\right)$ and signaling transducer and activator of transcription 3 (STAT3; FDR $<4.57 \times 10^{-7}$ ) were enriched for the downregulated genes. After analysis, the upregulated genes did not enrich any TF. The present study then analyzed the enriched TF binding motifs in the promoters of DEGs. DEG promoters were revealed to contain a number of G-rich motifs, and would be bound by activator protein-2 (AP-2), and other factors such as basic helix-loop-helix protein (bHLH) and Cys2-His2 zinc finger (C2H2 ZF; Table VI).

The predicted target genes for numerous miRNAs were then analyzed. As presented in Table VII, it was identified that miR-30e-5p, miR-181a-5p and miR-340-5p were significantly enriched target genes for the downregulated genes. In addition, miR-466d-5p and miR-4661-5p were significantly enriched target genes for the upregulated DEGs.

GSEA. To detect KEGG pathways that were differentially enriched in the PI and RE groups, GSEA was conducted between the PI and RE groups. The most significantly enriched signaling pathways were selected based on their NES (Fig. 6 and Table VIII). As presented in Fig. 6, 'toll like receptor (TLR) signaling', 'nod like receptor (NLR) signaling', 'pathogenic Escherichia coli infection', 'cytokine-cytokine receptor interaction' and 'Janus kinase (JAK)/STAT signaling' were differentially enriched in the PI group. Furthermore, 'aminoacyl tRNA biosynthesis', 'valine leucine and isoleucine degradation', 'one carbon pool by folate' and 'oxidative phosphorylation' were differentially enriched in the RE group.

\section{Discussion}

Sepsis is a clinically common and refractory critical illness. As a serious complication of sepsis, SA-AKI is the most common type of AKI in the clinic, with a high mortality rate (8). At present, owing to the lack of specific and effective means of SA-AKI prevention and treatment, it is of critical importance to study the pathophysiological mechanism and investigate new therapeutic targets in SA-AKI. Therefore, RNA-Seq was used in the present study to investigate gene expression profiles
Table VI. TF motifs enriched in gene promoters (300 bp) of the upregulated or downregulated genes.

\begin{tabular}{|c|c|c|c|}
\hline Motif & $\mathrm{TF}$ & TF family & FDR \\
\hline GCCTCAGG & Tcfap2a & AP-2 & $<0.001$ \\
\hline TCGCCTCAGG & Tcfap $2 b$ & AP-2 & $<0.001$ \\
\hline GCCCGAGGC & Tcfap2c & AP-2 & $<0.001$ \\
\hline GCCTGAGG & Tcfap2e & AP-2 & $<0.001$ \\
\hline CACGCG & Hes 1 & bHLH & $<0.001$ \\
\hline CGCGTG & Hes7 & bHLH & $<0.001$ \\
\hline CGTGC & Sohlh2 & bHLH & $<0.001$ \\
\hline CACGTG & Tcfl5 & bHLH & $<0.001$ \\
\hline GGGGGCGG & Sp1 & $\mathrm{C} 2 \mathrm{H} 2 \mathrm{ZF}$ & $<0.001$ \\
\hline GGGGGGTC & Glis2 & $\mathrm{C} 2 \mathrm{H} 2 \mathrm{ZF}$ & $<0.001$ \\
\hline GGGGC & Plagl1 & $\mathrm{C} 2 \mathrm{H} 2 \mathrm{ZF}$ & $<0.001$ \\
\hline GGGGGCGG & Sp4 & $\mathrm{C} 2 \mathrm{H} 2 \mathrm{ZF}$ & $<0.001$ \\
\hline GGGGGCGG & $\mathrm{Sp} 4$ & $\mathrm{C} 2 \mathrm{H} 2 \mathrm{ZF}$ & $<0.001$ \\
\hline GGCC & Zfp711 & $\mathrm{C} 2 \mathrm{H} 2 \mathrm{ZF}$ & $<0.001$ \\
\hline GGGG & Zfp202 & $\mathrm{C} 2 \mathrm{H} 2 \mathrm{ZF}$ & $<0.001$ \\
\hline GGGCGTG & Klf7 & $\mathrm{C} 2 \mathrm{H} 2 \mathrm{ZF}$ & $<0.001$ \\
\hline GGGGGC & Zbtb7b & $\mathrm{C} 2 \mathrm{H} 2 \mathrm{ZF}$ & $<0.001$ \\
\hline CACAGCGGG & Zic1 & $\mathrm{C} 2 \mathrm{H} 2 \mathrm{ZF}$ & $<0.001$ \\
\hline TGCGGG & Zbtb1 & $\mathrm{C} 2 \mathrm{H} 2 \mathrm{ZF}$ & $<0.001$ \\
\hline CGTGGGCG & Egr3 & $\mathrm{C} 2 \mathrm{H} 2 \mathrm{ZF}$ & $<0.001$ \\
\hline
\end{tabular}

B, Upregulated genes

\begin{tabular}{llll}
\hline Motif & \multicolumn{1}{c}{ TF } & TF family & FDR \\
\hline GGGGGCGG & Sp1 & C2H2 ZF & $<0.001$ \\
GGGGGCGG & Sp4 & C2H2 ZF & $<0.001$ \\
GGGGGCGG & Sp4 & C2H2 ZF & $<0.001$ \\
GGGCGTG & Klf7 & C2H2 ZF & $<0.001$ \\
TGCGGG & Zbtb1 & C2H2 ZF & $<0.001$ \\
GGGCG & Klf8 & C2H2 ZF & $<0.001$ \\
GGGGGGG & Zfp740 & C2H2 ZF & $<0.001$ \\
GGGGGG & Zfp740 & C2H2 ZF & $<0.001$ \\
CGCGC & Zfp161 & C2H2 ZF & $<0.001$ \\
CG & Cxxc1 & CxxC & $<0.001$ \\
CG & Kdm2b & CxxC & $<0.001$ \\
GCGC & E2f3 & E2F & $<0.001$ \\
GGCGC & E2f2 & E2F & $<0.001$ \\
GTGGGGGCGGGAG & E2f3 & E2F & $<0.001$ \\
GGGGGCGGGGC & Sp2 & C2H2 ZF & $<0.001$ \\
GGGCGGGGC & Klf5 & C2H2 ZF & $<0.001$ \\
GGGGGGGGGCC & Patz1 & C2H2 ZF & $<0.001$ \\
CACAGCGGGGGGTC & Zic4 & C2H2 ZF & $<0.001$ \\
GTGGGGGGG & Zfp740 & C2H2 ZF & $<0.001$ \\
CACAGCGGGGGGTC & Zic3 & C2H2 ZF & $<0.001$ \\
\hline
\end{tabular}

$\mathrm{TF}$, transcription factor; FDR, false discovery rate.

related to mice with SA-AKI that exhibited persistent injury or recovery. The current study identified some prognosis-related 
Table VII. Enriched miRNA target genes of the upregulated and downregulated genes.

\begin{tabular}{|c|c|c|}
\hline $\begin{array}{l}\text { Adjusted } \\
\text { P-value }\end{array}$ & Genes (n) & Target miRNAs \\
\hline$<0.001$ & 71 & MiRTarBase:mmu-miR-30e-5p \\
\hline$<0.001$ & 80 & MiRTarBase:mmu-miR-181a-5p \\
\hline$<0.001$ & 93 & MiRTarBase:mmu-miR-340-5p \\
\hline$<0.001$ & 73 & MiRTarBase:mmu-miR-124-3p \\
\hline$<0.001$ & 21 & MiRTarBase:mmu-miR-155-5p \\
\hline$<0.001$ & 14 & MiRTarBase:mmu-miR-338-5p \\
\hline$<0.001$ & 57 & MiRTarBase:mmu-miR-129-5p \\
\hline$<0.001$ & 81 & MiRTarBase:mmu-miR-329-3p \\
\hline$<0.001$ & 62 & MiRTarBase:mmu-miR-466i-3p \\
\hline$<0.001$ & 53 & MiRTarBase:mmu-miR-19b-3p \\
\hline$<0.001$ & 33 & MiRTarBase:mmu-miR-377-3p \\
\hline$<0.001$ & 25 & MiRTarBase:mmu-miR-342-3p \\
\hline 0.003 & 71 & MiRTarBase:mmu-miR-15a-5p \\
\hline 0.004 & 52 & MiRTarBase:mmu-let-7b-5p \\
\hline
\end{tabular}

B, Upregulated genes

\begin{tabular}{|c|c|c|}
\hline $\begin{array}{l}\text { Adjusted } \\
\text { P-value }\end{array}$ & Genes (n) & Target miRNAs \\
\hline$<0.001$ & 84 & MiRTarBase:mmu-miR-466d-5p \\
\hline$<0.001$ & 84 & MiRTarBase:mmu-miR-4661-5p \\
\hline$<0.001$ & 30 & MiRTarBase:mmu-miR-150-5p \\
\hline$<0.001$ & 67 & MiRTarBase:mmu-miR-1195 \\
\hline$<0.001$ & 27 & MiRTarBase:mmu-miR-5127 \\
\hline$<0.001$ & 69 & MiRTarBase:mmu-miR-3089-5p \\
\hline$<0.001$ & 29 & MiRTarBase:mmu-miR-466n-5p \\
\hline$<0.001$ & 51 & MiRTarBase:mmu-miR-466m-3p \\
\hline$<0.001$ & 41 & MiRTarBase:mmu-miR-3064-5p \\
\hline 0.003 & 45 & MiRTarBase:mmu-miR-665-3p \\
\hline 0.003 & 61 & MiRTarBase:mmu-miR-129-5p \\
\hline
\end{tabular}

genes, transcription factors, miRNAs and pathways associated with the differential gene expression profiles, including metabolic process, the transcription factor RELA and miR-30e-5p.

$\kappa$-means clustering was used to gain insight into the molecular pathways underlying different gene expression patterns. This divided the top 2,000 DEGs into four clusters. Genes in cluster B were upregulated in the RE group, and this cluster was strongly enriched in genes related to cellular metabolic processes, such as lipid metabolism and fatty acid metabolism. It has been reported that lipoproteins play an important role in preventing infection and inflammation (13). All lipoproteins can bind and neutralize toxic bacterial substances to modulate cytokine production during the inflammatory response, which can weaken the host response (14-16). Therefore, low lipoprotein levels may damage the innate immune response to endotoxins, leading to a deterioration of the systemic inflammatory cascade $(15,17-20)$. Svahn et al (21) reported that dietary omega-3 fatty acids can increase sepsis survival and stimulate the immune system. Omega-3 fatty acids can be metabolized into a novel substance group termed resolvins (Rv) (22). It has been suggested that treatment with RvD1 and RvD2 can increase the survival of patients with sepsis (22-24). One putative mechanism underlying this process may be the combination of an increased frequency of neutrophils and precursor cells in bone marrow and restored phagocytotic capacity (21).

Genes in cluster $\mathrm{C}$ were highly upregulated in the PI group, and this cluster was associated with cytokines, cell death and apoptosis. Multiple pathways related to cell death are involved in sepsis due to the pro-inflammatory, anti-inflammatory, coagulation and complement systems. Apoptosis is a natural process of cell death, which is genetically programmed and plays a crucial role in normal physiology and in the pathophysiology of sepsis $(25,26)$. Apoptosis can be initiated by pro-inflammatory cytokines, such as tumor necrosis factor $\alpha$ (TNF- $\alpha$ ), interleukin (IL)-1 and IL-6 (27). It has been suggested that prevention of cell apoptosis can improve survival in animal models of sepsis and endotoxemia (27).

With FDR $<0.1$ and FC $>2$ as the cutoff, 5,402 DEGs (2,256 downregulated and 3,146 upregulated genes) were identified, which have potential to be novel regulators and may play a role in the pathophysiological mechanism underlying SA-AKI development. To further understand the molecular pathways, enrichment analysis of the DEGs was performed. For the GO Biological Process analysis, consistent with the results of k-means clustering analysis, it was identified that the upregulated genes were predominantly involved in cellular metabolic processes. Furthermore, the downregulated genes were associated with cell response to stress, cytokine production, apoptosis and cell death.

As for the GO Cellular Component analysis, it was identified that upregulated DEGs in the RE group were enriched in the mitochondrion, cytoskeleton and centrosome. Mitochondria are cytoplasmic organelles with a double phospholipid membrane that generate energy via oxidative phosphorylation (28). Mitochondria are also associated with calcium homeostasis, intracellular reactive oxygen species (ROS) generation and cell signaling functions (29,30). In response to sepsis, the inflammatory cytokines of the innate immune response, including TNF- $\alpha$, IL-1 and IL-6, can promote mitochondrial permeability transition, inhibit oxidative phosphorylation and improve ROS production $(31,32)$. It has been demonstrated that mitochondrial dysfunction plays a critical role in SA-AKI (33). However, further studies are still needed to clarify the molecular mechanism underlying mitochondrial dysfunction in SA-AKI.

The cytoskeleton is a necessary dynamic structure for cells that plays an important role in regulating cell permeability under physiological and pathophysiological conditions (34). The cytoskeleton pathway is a known modulator of endothelial barrier function and microvascular permeability (35). It is well known that an inflammatory stimulus can lead to the development of capillary leak and tissue edema due to cytoskeletal rearrangement, which is a characteristic feature of sepsis; its development is one of the 
Table VIII. Differentially expressed pathways enriched in the PI and RE mice according to gene set enrichment analysis.

\begin{tabular}{lll}
\hline A, PI group & & \\
\hline Pathway name & NES & Nominal P-value \\
\hline Toll_Like_Receptor_Signaling & 2.90 & $<0.001$ \\
Nod_Like_Receptor_Signaling & 2.81 & $<0.001$ \\
Pathogenic_Escherichia_Coli_Infection & 2.74 & $<0.001$ \\
Cytokine_Cytokine_Receptor_Interaction & 2.60 & $<0.001$ \\
JAK_STAT_Signaling_Pathway & 2.59 & $<0.001$ \\
RIG_I_Like_Receptor_Signaling & 2.53 & $<0.001$ \\
Leishmania_Infection & 2.51 & $<0.001$ \\
Small_Cell_Lung_Cancer & 2.45 & $<0.001$ \\
Cytosolic_Dna_Sensing & 2.42 & $<0.001$ \\
Chronic_Myeloid_Leukemia & 2.33 & $<0.001$ \\
\hline
\end{tabular}

B, RE group

\begin{tabular}{lrrr}
\hline Pathway name & NES & Nominal P-value & FDR value \\
\hline Aminoacyl_tRNA_Biosynthesis & -1.75 & 0.001 & 0.095 \\
Valine_Leucine_And_Isoleucine_Degradation & -1.73 & $<0.001$ & 0.065 \\
One_Carbon_Pool_By_Folate & -1.70 & 0.006 & 0.065 \\
Oxidative_Phosphorylation & -1.69 & $<0.001$ & 0.053 \\
\hline
\end{tabular}

PI, persistent injury; RE, recovery; NES, normalized enrichment score.

causes of organ dysfunction in sepsis (36). A meta-analysis of transcriptomic data demonstrated that the cytoskeletal pathway was upregulated in sepsis patients compared with controls (37). Previous studies have found that the breakdown in blood/endothelial barrier function plays a vital role in the pathogenesis and organ function and could be a new therapeutic target for sepsis $(38,39)$.

Cytokine production is a milestone in the immune response during inflammation and is associated with mortality during sepsis (40). Vertii et al (41) found that centrosome integrity is critical for cytokine production. Impaired cytokine production may predispose the host to infection.

For the GO Molecular Function analysis, it was confirmed that the downregulated genes in the RE group were enriched in enzyme binding and receptor binding. Furthermore, the upregulated genes in the RE group were enriched in transporter activity and active transmembrane transporter activity. This could indicate that transporter activity and active transmembrane transporter activity may be involved in sepsis recovery. The Slc7a7 gene provides instructions for the production of a protein termed $\mathrm{y}+\mathrm{L}$ amino acid transporter 1 ( $\mathrm{y}+\mathrm{LAT}-1)$, which is involved in transporting certain protein building blocks (amino acids), namely, lysine, arginine and ornithine. The transport of amino acids from the small intestines and kidneys to the rest of the body is necessary for the body to utilize proteins. The $y+$ LAT-1 protein forms one part (the light subunit) of a complex called the heterodimeric cationic amino acid transporter. This subunit is responsible for binding to the amino acids that are transported (42). However, whether the Slc7a7 gene is involved in the recovery of sepsis requires further study.

The present study identified that the TF target genes RELA and STAT3 were significantly associated with the downregulated genes in the RE group. RELA is a REL-associated protein involved in $\mathrm{NF}-\kappa \mathrm{B}$ heterodimer formation, nuclear translocation and activation (43). As a nuclear transcription factor of various cells, NF- $\kappa \mathrm{B}$ plays a crucial role in the coordination of innate and adaptive immune responses in sepsis (44). $\mathrm{NF}-\kappa \mathrm{B}$ binds with I $\mathrm{B}-\alpha$ and forms a p65/p60 dimer in the cytoplasm in physiological conditions (45). In stress, IкB- $\alpha$ isolates $\mathrm{NF}-\kappa \mathrm{B}$ and is transferred to the nucleus, where it binds proinflammatory cytokines and promotes the gene transcription of TNF- $\alpha$ and IL- 6 to further activate NF- $\kappa$ B to expand the inflammatory response (46). STAT3 is a member of the STAT protein family and can mediate the expression of various genes that stimulate cells. In response to cytokines and growth factors, STAT3 can be phosphorylated by receptor-related JAK and translocate to the cell nucleus where it acts as a transcriptional activator (47). The STAT3 pathway plays an important role in inflammatory signaling cascades and its activation can play a crucial role in host-bacterial interactions (48). Cytokines, such as IL-6 and IL-10, can cause the phosphorylation of the tyrosine and serine residues of STAT3 via JAK. Activated STAT3 can in turn translocate into the nucleus where it binds to specific promotor sequences and regulate the transcription of target genes (49). Zhuo et al (24) demonstrated that RvD1 may improve survival and attenuate the degree of lung inflammation of septic mice by suppressing 
A

Enrichment plot:

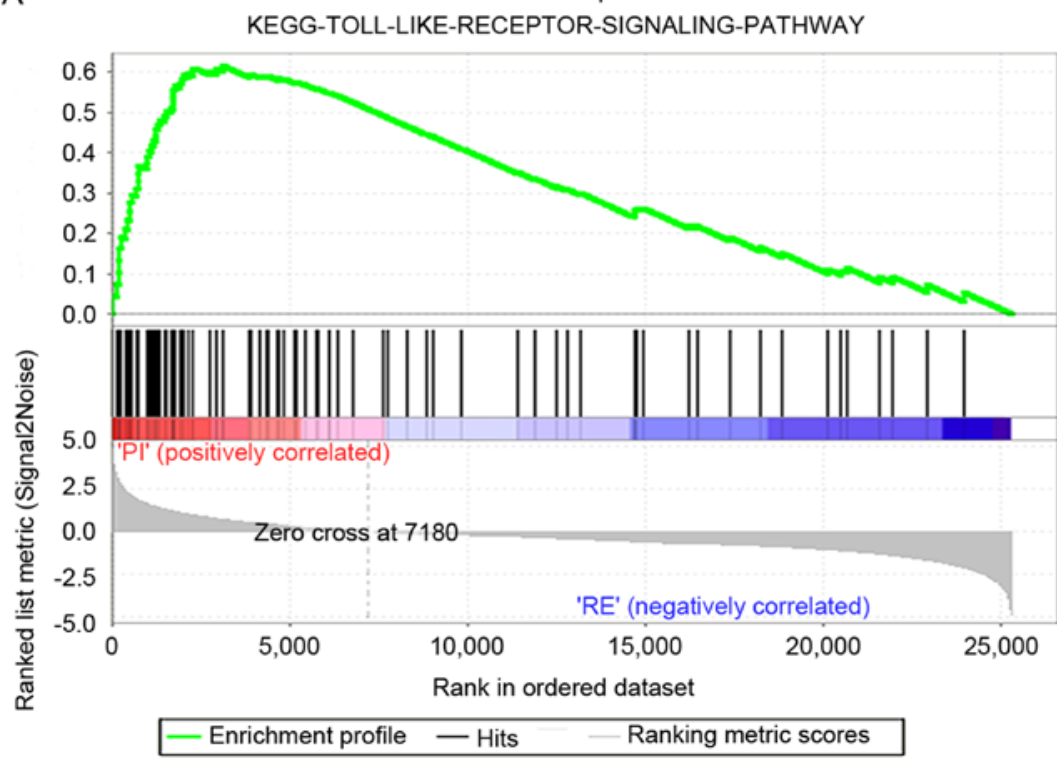

B

Enrichment plot:

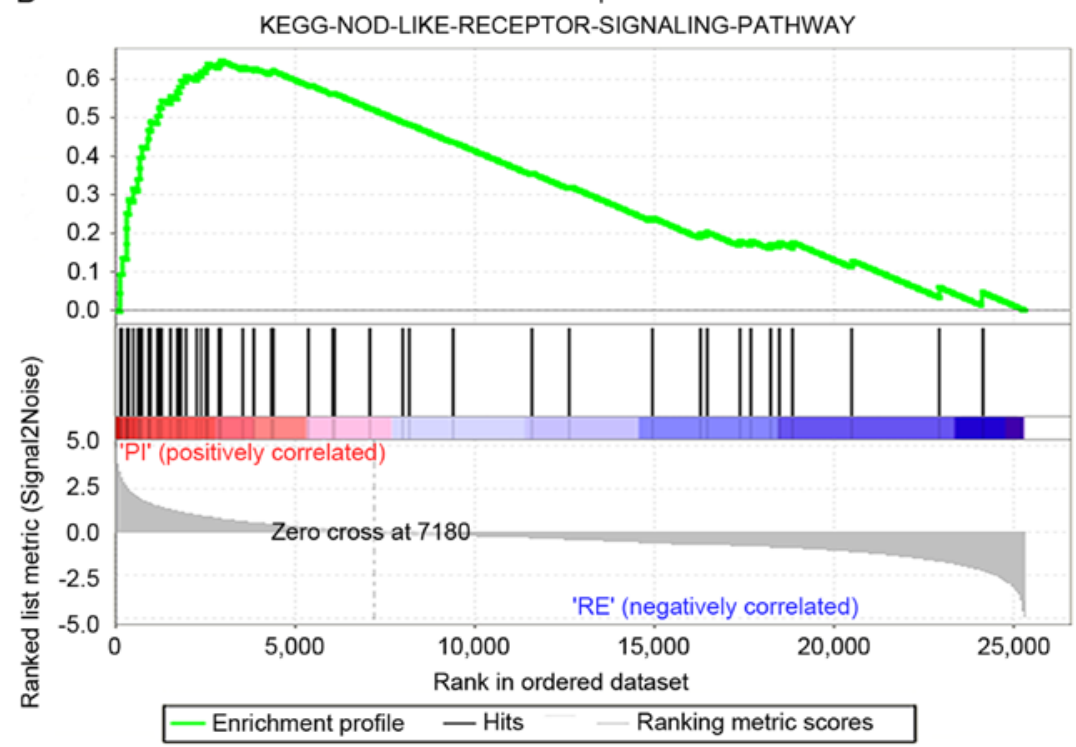

C

Enrichment plot:

KEGG-PATHOGENIC_ESCHERICHIA_COLI_INFECTION

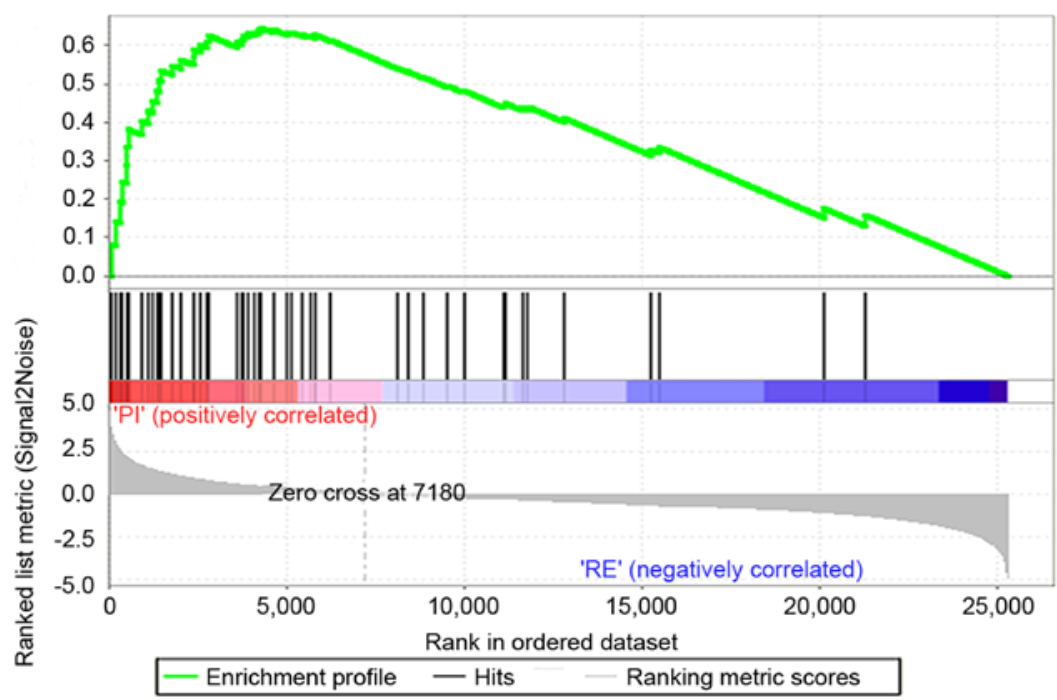

Figure 6. Enrichment plots from GSEA. GSEA results showing that (A) 'Toll-like receptor signaling', (B) 'NOD-like receptor signaling', (C) 'Pathogenic Escherichia coli infection' were differentially enriched in the PI group. 


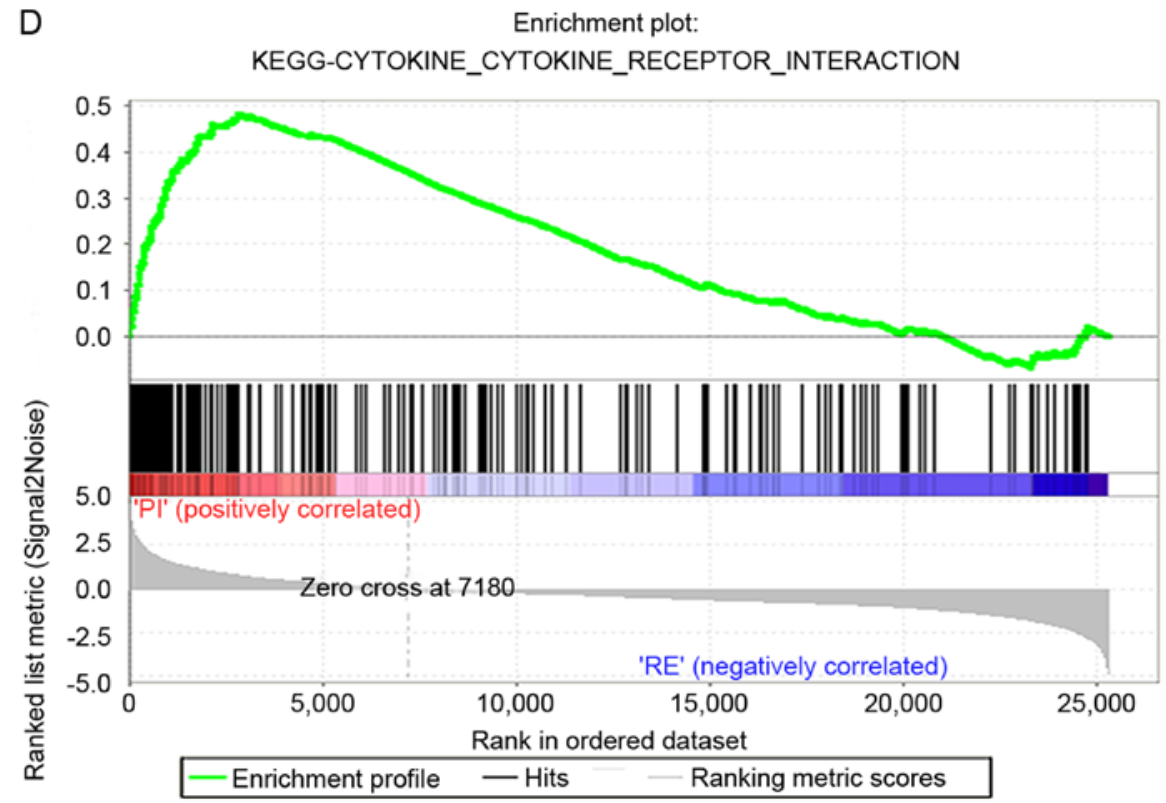

E Enrichment plot: KEGG-JAK_STAT_SIGNALING_PATHWAY

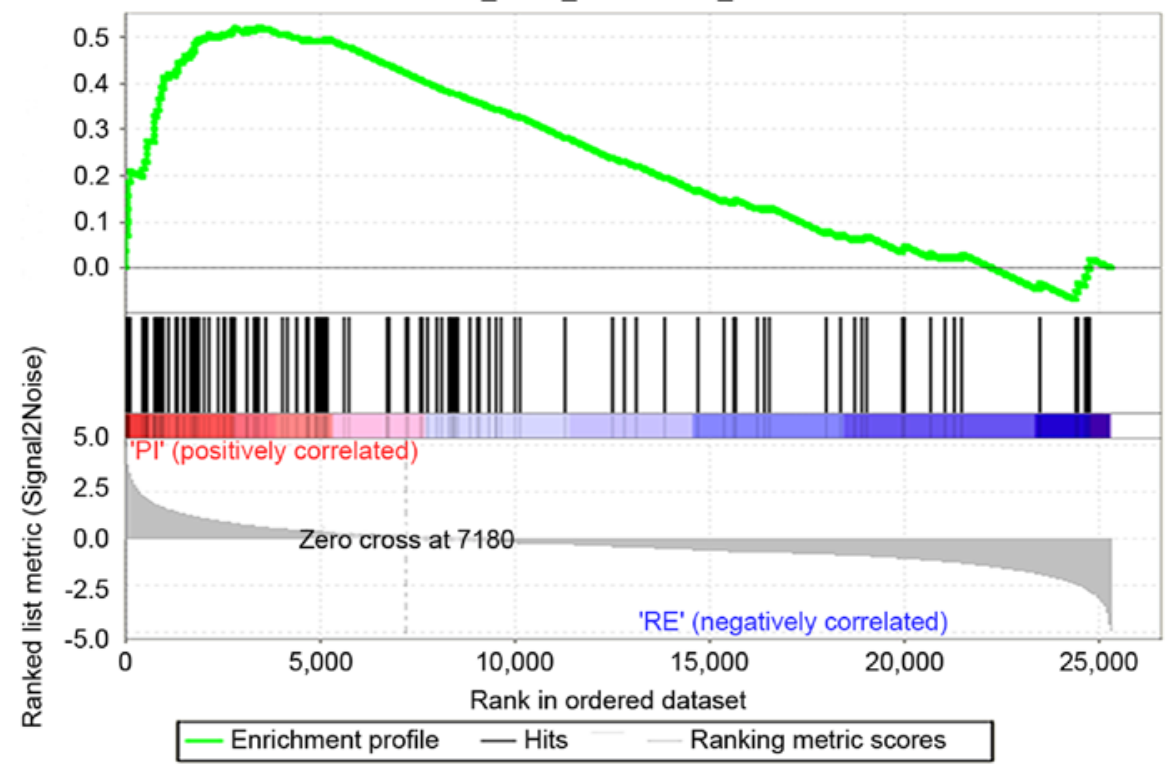

Figure 6. Continued. Enrichment plots from GSEA. GSEA results showing that (D) 'Cytokine-cytokine receptor interaction' and (E) 'JAK/STAT signaling' were differentially enriched in the PI group.

STAT3 and NF- $\mathrm{B}$ expression through a mechanism that is partly dependent on SIRT1.

TF binding motifs (TFBMs) have conserved DNA sequence elements in their promoter regions. TFBMs can act as binding sites for transcription factors and coordinate the expression of genes in the promoter regions that they appear (50). As TFs typically bind to DNA at sites matching specific sequences motifs, knowledge of the motifs for a TF will be useful to determine a potential binding site of the TF (51). The present study detected that the DEG promoters were overrepresented with numerous G-rich motifs that are bound by AP-2 and other factors, such as bHLH and C2H2 ZF. AP-2 transcription factors (TFAP-2) constitute a family of closely related and evolutionarily conserved proteins that bind to the DNA consensus sequence $\mathrm{GCCN}_{3} \mathrm{GGC}$ and stimulate target gene transcription (52). AP-2 consists of five different proteins in human and mouse: AP- $2 \alpha$, AP- $2 \beta$, AP- $2 \gamma, \mathrm{AP}-2 \delta$ and AP- $2 \varepsilon$ (53). A previous study found that various inflammatory cytokines and prostaglandins can induce the expression of TFAP-2, which in turn causes aberrant activation of genes associated with hyperproliferation of mesangial cells and nephrosclerosis (54). Knocking down TFAP2A significantly decreases TF binding and the gene expression of collagen IV, which may play a critical role in diabetic nephropathy (55).

Our study demonstrated that miR-30e-5p, miR-181a-5p and miR-340-5p were significantly enriched target genes for the downregulated genes, and miR-466d-5p and miR-4661-5p were significantly enriched target genes for the upregulated DEGs. miRNAs and TFs share a common link as two vital gene regulatory molecules in multicellular organisms (56). miRNAs 
$\mathrm{F}$

Enrichment plot:

KEGG-AMINOACYL_TRNA_BIOSYNTHESIS

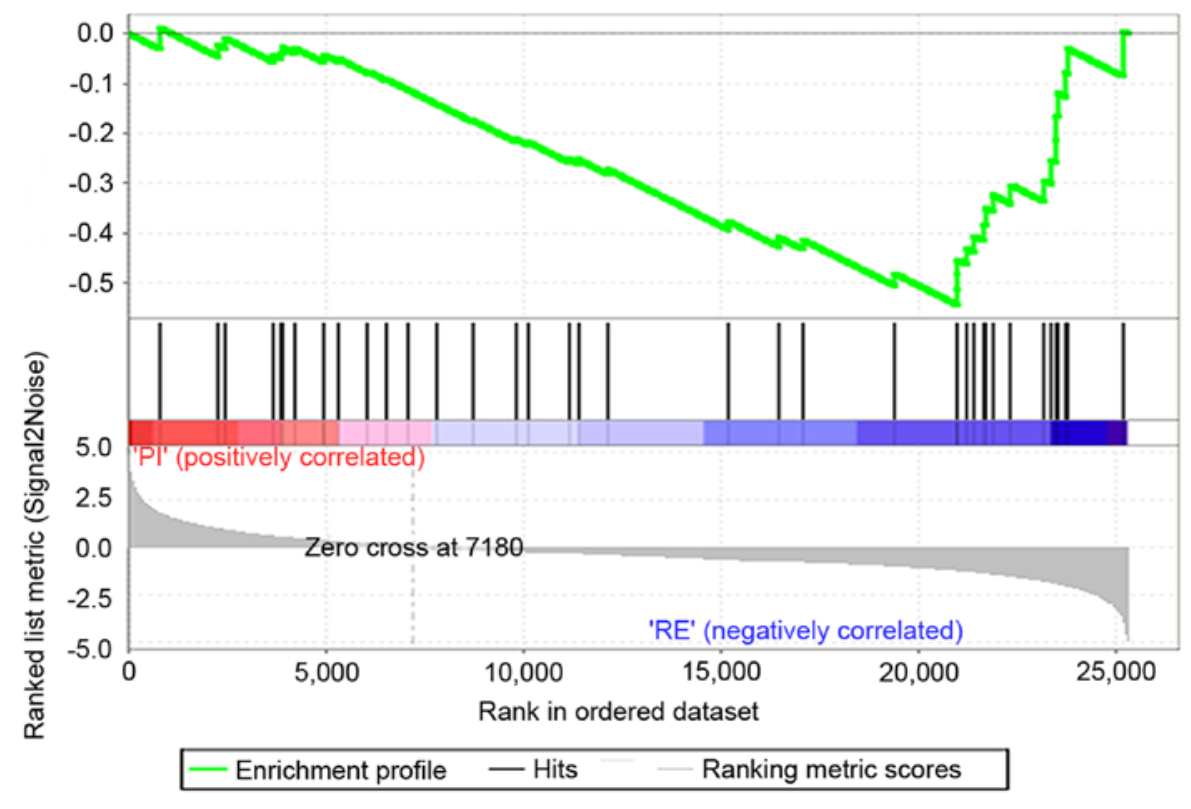

G

Enrichment plot:

KEGG-VALINE_LEUCINE_AND_ISOLEUCINE_DEGRADATION

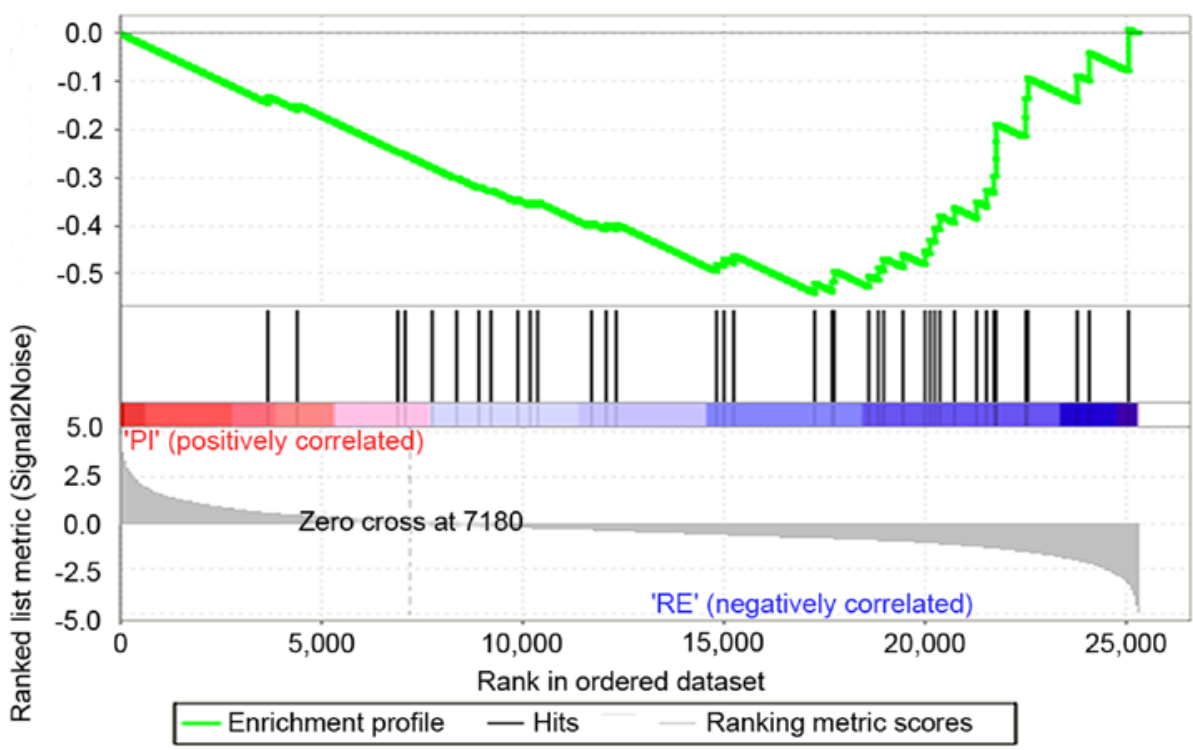

Figure 6. Continued. Enrichment plots from GSEA. GSEA results showing that (F) 'Aminoacyl tRNA biosynthesis', (G) 'Valine leucine and isoleucine degradation' were differentially enriched in the RE group.

are a family of small non-coding RNAs that modulate gene expression in a sequence-specific manner, and as a result they regulate numerous cellular processes at the post-transcriptional level (57). miR-30e-5p is a member of the miR-30 family, and plays a critical role in renal development and maintaining renal function (58). Sun et al (59) reported that the circulating miR-30e-5p level is significantly elevated in contrast-induced AKI. This may be associated with the pathophysiology of AKI. The miR-181 family has been demonstrated to control inflammation under physiological and pathological conditions by modulating various key aspects of growth, development and activation (60). A previous study demonstrated that miR-340 can inhibit cell proliferation, tumor migration and invasion by targeting JAK via the JAK1/STAT3 signaling pathway (61). It has been indicated that miR-4661 may to be a negative regulator of multiple proinflammatory cytokines, such as interferons and IL-10 $(62,63)$. These miRNAs may be potential therapeutic targets for SA-AKI.

The innate immune system is the first line of defense against microbial invasion, relying on pattern recognition receptors to recognize external pathogenic microorganisms and then remove them (64). TLRs and NLRs are important receptors that mediate immune recognition by recognizing pathogen-associated molecular patterns derived from various microbes. These processes are bridges between innate immunity and adaptive immunity (65). 
$\mathrm{H}$

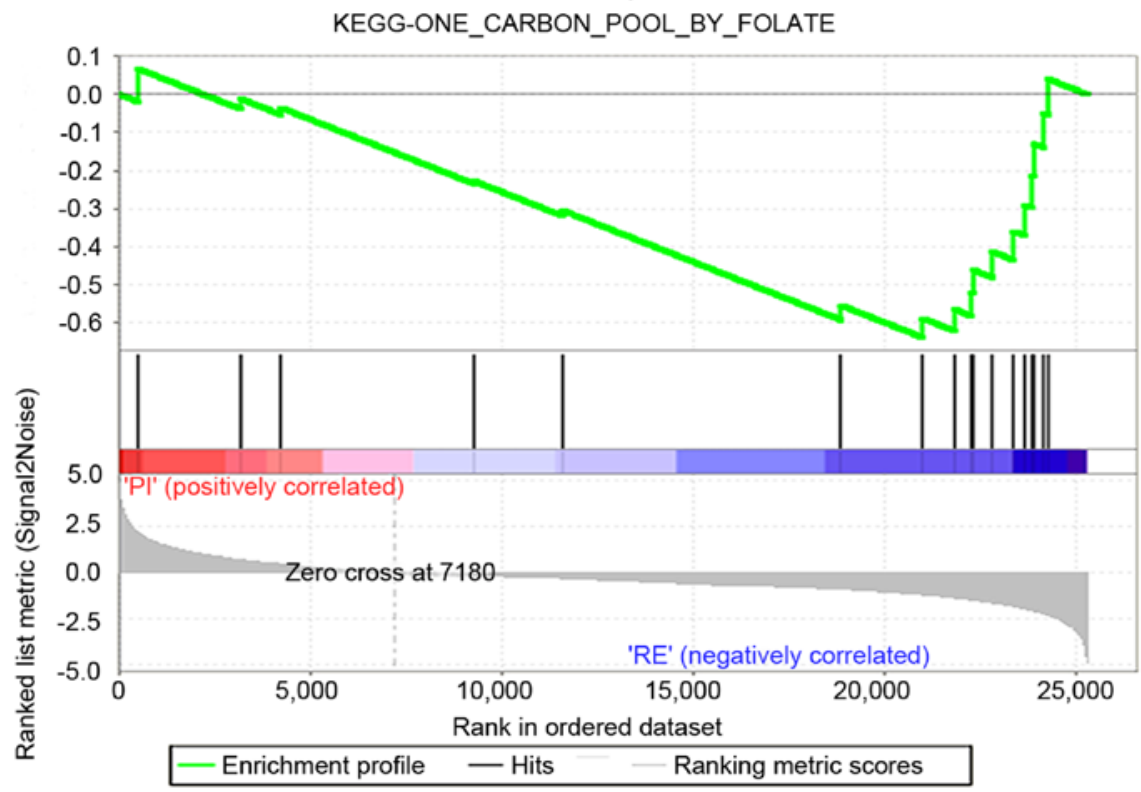

I

Enrichment plot:

KEGG-OXIDATIVE_PHOSPHORYLATION

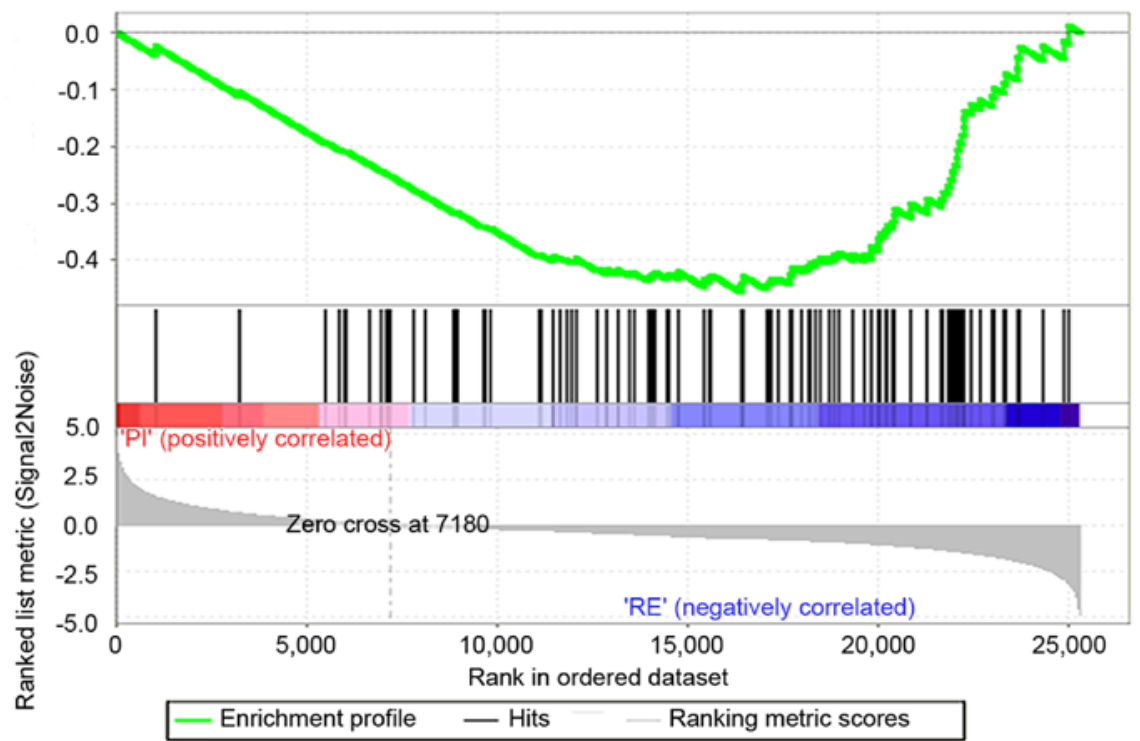

Figure 6. Continued. Enrichment plots from GSEA. GSEA results showing that (H) 'One carbon pool by folate' and (I) 'Oxidative phosphorylation' were differentially enriched in the RE group. GSEA, gene set enrichment analysis; RE, recovery; PI, persistent injury.

In conclusion, the present study examined gene expression profiles of a SA-AKI model that exhibited persistent renal injury or renal recovery using RNA-Seq. A number of prognosis-related genes, transcription factors, miRNAs and pathways were identified to be associated with the differential expression profile, including metabolic process, the transcription factor RELA and miR-30e-5p, which provide some basis for future experimental studies. Further validation, particularly in human tissues, may provide a more comprehensive understanding of the underlying pathophysiology during SA-AKI.

\section{Acknowledgements}

The authors wish to thank Dr Wanling Yang (Department of Pediatrics and Adolescent Medicine, Li Ka Shing Faculty of Medicine, University of Hong Kong, Hong Kong, SAR, P.R. China) for providing constructive instructions and comments on the design of the present study.

\section{Funding}

This work was supported by a grant from the National Nature Science Foundation of China (grant no. 81670621) and the Nature Science Foundation of Zhejiang Province (grant no. LY16H050001).

\section{Availability of data and materials}

The datasets used and/or analysed during the current study are available from the corresponding author on reasonable request. 


\section{Authors' contributions}

YY conceived the study, participated in its design and coordination, and drafted the manuscript. JY participated in the experiments and bioinformatics analysis, and contributed to drafting the manuscript. BW performed all statistical analysis. FH participated in its design and coordination, drafted the manuscript and revised the manuscript for important intellectual content. JC conceived the study and revised the manuscript important intellectual content. All authors read and approved the final manuscript.

\section{Ethics approval and consent to participate}

All protocols of this study were approved by the Animal Ethics Committee of the First Affiliated Hospital, College of Medicine, Zhejiang University, Hangzhou, Zhejiang (approval ID. 2016160; 25 February 2016), which follows the institutional guidelines.

\section{Patient consent for participation}

Not applicable.

\section{Competing interests}

The authors declare that they have no competing interests.

\section{References}

1. Singer M, Deutschman CS, Seymour CW, Shankar-Hari M, Annane D, Bauer M, Bellomo R, Bernard GR, Chiche JD, Coopersmith CM, et al: The third international consensus definitions for sepsis and septic shock (Sepsis-3). JAMA 315: 801-810, 2016.

2. Kellum JA and Lameire N; KDIGO AKI Guideline Work Group: Diagnosis, evaluation, and management of acute kidney injury: A KDIGO summary (Part 1). Crit Care 17: 204, 2013.

3. Hoste EA, Lameire NH, Vanholder RC, Benoit DD, Decruyenaere JM and Colardyn FA: Acute renal failure in patients with sepsis in a surgical ICU: Predictive factors, incidence, comorbidity, and outcome. J Am Soc Nephrol 14: 1022-1030, 2003.

4. Uchino S, Kellum JA, Bellomo R, Doig GS, Morimatsu H, Morgera S, Schetz M, Tan I, Bouman C, Macedo E, et al: Acute renal failure in critically ill patients: A multinational, multicenter study. JAMA 294: 813-818, 2005.

5. Morrell ED, Kellum JA, Pastor-Soler NM and Hallows KR: Septic acute kidney injury: Molecular mechanisms and the importance of stratification and targeting therapy. Crit Care 18: 501, 2014.

6. Bagshaw SM, Uchino S, Bellomo R, Morimatsu H, Morgera S, Schetz M, Tan I, Bouman C, Macedo E, Gibney N, et al: Septic acute kidney injury in critically ill patients: Clinical characteristics and outcomes. Clin J Am Soc Nephrol 2: 431-439, 2007.

7. Bouchard J, Acharya A, Cerda J, Maccariello ER, Madarasu RC, Tolwani AJ, Liang X, Fu P, Liu ZH and Mehta RL: A prospective international multicenter study of AKI in the intensive care unit. Clin J Am Soc Nephrol 10: 1324-1331, 2015.

8. Poston JT and Koyner JL: Sepsis associated acute kidney injury. BMJ 364: k4891, 2019.

9. Tran M, Tam D, Bardia A, Bhasin M, Rowe GC, Kher A, Zsengeller ZK, Akhavan-Sharif MR, Khankin EV, Saintgeniez M, et al: PGC-1 $\alpha$ promotes recovery after acute kidney injury during systemic inflammation in mice. J Clin Invest 121: 4003-4014, 2011.

10. Stark R, Grzelak M and Hadfield J: RNA sequencing: The teenage years. Nat Rev Genet 20: 631-656, 2019.
11. Ge SX, Son EW and Yao R: iDEP: An integrated web application for differential expression and pathway analysis of RNA-Seq data. BMC Bioinformatics 19: 534, 2018.

12. Subramanian A, Tamayo P, Mootha VK, Mukherjee S, Ebert BL, Gillette MA, Paulovich A, Pomeroy SL, Golub TR, Lander ES and Mesirov JP: Gene set enrichment analysis: A knowledge-based approach for interpreting genome-wide expression profiles. Proc Natl Acad Sci USA 102: 15545-15550, 2005.

13. Goldstein B, Giroir B and Randolph A; International Consensus Conference on Pediatric Sepsis: International pediatric sepsis consensus conference: Definitions for sepsis and organ dysfunction in pediatrics. Pediatr Crit Care Med 6: $2-8,2005$.

14. Wu A, Hinds CJ and Thiemermann C: High-density lipoproteins in sepsis and septic shock: Metabolism, actions, and therapeutic applications. Shock 21: 210-221, 2004.

15. Murch O, Collin M, Hinds CJ and Thiemermann C: Lipoproteins in inflammation and sepsis. I. Basic science. Intensive Care Med 33: 13-24, 2007.

16. Vyroubal P, Chiarla C, Giovannini I, Hyspler R, Ticha A, Hrnciarikova D and Zadak Z: Hypocholesterolemia in clinically serious conditions-review. Biomed Pap Med Fac Univ Palacky Olomouc Czech Repub 152: 181-189, 2008.

17. Tsai MH, Peng YS, Chen YC, Lien JM, Tian YC, Fang JT, Weng HH, Chen PC, Yang CW and Wu CS: Low serum concentration of apolipoprotein A-I is an indicator of poor prognosis in cirrhotic patients with severe sepsis. J Hepatol 50: 906-915, 2009.

18. Chenaud C, Merlani PG, Roux-Lombard P, Burger D, Harbarth S, Luyasu S, Graf JD, Dayer JM and Ricou B: Low apolipoprotein A-I level at intensive care unit admission and systemic inflammatory response syndrome exacerbation. Crit Care Med 32: 632-637, 2004.

19. Barlage S, Gnewuch C, Liebisch G, Wolf Z, Audebert FX, Glück T, Fröhlich D, Krämer BK, Rothe G and Schmitz G: Changes in HDL-associated apolipoproteins relate to mortality in human sepsis and correlate to monocyte and platelet activation. Intensive Care Med 35: 1877-1885, 2009.

20. Bonville DA, Parker TS, Levine DM, Gordon BR, Hydo LJ, Eachempati SR and Barie PS: The relationships of hypocholesterolemia to cytokine concentrations and mortality in critically ill patients with systemic inflammatory response syndrome. Surg Infect (Larchmt.) 5: 39-49, 2004.

21. Svahn SL, Ulleryd MA, Grahnemo L, Ståhlman M, Borén J, Nilsson S, Jansson JO and Johansson ME: Dietary omega-3 fatty acids increase survival and decrease bacterial load in mice subjected to staphylococcus aureus-induced sepsis. Infect Immun 84: 1205-1213, 2016.

22. Spite M, Norling LV, Summers L, Yang R, Cooper D, Petasis NA, Flower RJ, Perretti M and Serhan CN: Resolvin D2 is a potent regulator of leukocytes and controls microbial sepsis. Nature 461: 1287-1291, 2009.

23. Chen F, Fan XH, Wu YP, Zhu JL, Wang F, Bo LL, Li JB, Bao R and Deng XM: Resolvin D1 improves survival in experimental sepsis through reducing bacterial load and preventing excessive activation of inflammatory response. Eur J Clin Microbiol Infect Dis 33: 457-464, 2014

24. Zhuo Y, Zhang S, Li C, Yang L, Gao H and Wang X: Resolvin D1 promotes SIRT1 expression to counteract the activation of STAT3 and NF- $\mathrm{BB}$ in mice with septic-associated lung injury. Inflammation 41: 1762-1771, 2018.

25. Kerr JF, Wyllie AH and Currie AR: Apoptosis: A basic biological phenomenon with wide-ranging implications in tissue kinetics. Br J Cancer 26: 239-257, 1972.

26. Ayala A, Perl M, Venet F, Lomas-Neira J, Swan R and Chung CS: Apoptosis in sepsis: Mechanisms, clinical impact and potential therapeutic targets. Curr Pharm Des 14: 1853-1859, 2008.

27. Harjai M, Bogra J, Kohli M and Pant AB: Is suppression of apoptosis a new therapeutic target in sepsis? Anaesth Intensive Care 41: 175-183, 2013.

28. Green DR, Galluzzi L and Kroemer G: Mitochondria and the autophagy-inflammation-cell death axis in organismal aging. Science 333: 1109-1112, 2011.

29. Galley HF: Oxidative stress and mitochondrial dysfunction in sepsis. Br J Anaesth 107: 57-64, 2011.

30. Rocha M, Herance R, Rovira S, Hernandez-Mijares A and Victor VM: Mitochondrial dysfunction and antioxidant therapy in sepsis. Infect Disord Drug Targets 12: 161-178, 2012.

31. Siskind LJ, Kolesnick RN and Colombini M: Ceramide channels increase the permeability of the mitochondrial outer membrane to small proteins. J Biol Chem 277: 26796-26803, 2002. 
32. Garcia-Ruiz C, Colell A, Mari M, Morales A and Fernandez-Checa JC: Direct effect of ceramide on the mitochondrial electron transport chain leads to generation of reactive oxygen species. Role of mitochondrial glutathione. J Biol Chem 272: 11369-11377, 1997.

33. Sureshbabu A, Patino E, Ma KC, Laursen K, Finkelsztein EJ, Akchurin O, Muthukumar T, Ryter SW, Gudas L, Choi AMK and Choi ME: RIPK3 promotes sepsis-induced acute kidney injury via mitochondrial dysfunction. JCI Insight 3: e98411, 2018.

34. Rodgers LS and Fanning AS: Regulation of epithelial permeability by the actin cytoskeleton. Cytoskeleton (Hoboken) 68: 653-660, 2011

35. Jacobson JR and Garcia JG: Novel therapies for microvascular permeability in sepsis. Curr Drug Targets 8: 509-514, 2007.

36. Gustot T: Multiple organ failure in sepsis: Prognosis and role of systemic inflammatory response. Curr Opin Crit Care 17: 153-159, 2011.

37. Ma J, Chen C, Barth AS, Cheadle C, Guan X and Gao L: Lysosome and cytoskeleton pathways are robustly enriched in the blood of septic patients: A meta-analysis of transcriptomic data. Mediators Inflamm 2015: 984825, 2015.

38. de Montmollin E and Annane D: Year in review 2010: Critical care-multiple organ dysfunction and sepsis. Crit Care 15: 236, 2011.

39. Goldenberg NM, Steinberg BE, Slutsky AS and Lee WL: Broken barriers: A new take on sepsis pathogenesis. Sci Transl Med 3: 88ps25, 2011.

40. Chousterman BG, Swirski FK and Weber GF: Cytokine storm and sepsis disease pathogenesis. Semin Immunopathol 39: 517-528, 2017.

41. Vertii A, Ivshina M, Zimmerman W, Hehnly H, Kant S and Doxsey S: The centrosome undergoes Plk1-independent interphase maturation during inflammation and mediates cytokine release. Dev Cell 37: 377-386, 2016.

42. Sperandeo MP, Andria G and Sebastio G: Lysinuric protein intolerance: Update and extended mutation analysis of the SLC7A7 gene. Hum Mutat 29: 14-21, 2008.

43. Lesina M, Wörmann SM, Morton J, Diakopoulos KN, Korneeva O, Wimmer M, Einwächter H, Sperveslage J, Demir IE, Kehl T, et al: RelA regulates CXCL1/CXCR2-dependent oncogene-induced senescence in murine Kras-driven pancreatic carcinogenesis. J Clin Invest 126: 2919-2932, 2016.

44. Chen Y, Guo L, Lang H, Hu X, Jing S, Luo M, Xu G and Zhou Z: Effect of a stellate ganglion block on acute lung injury in septic rats. Inflammation 41: 1601-1609, 2018

45. Zhu T, Wang DX, Zhang W, Liao XQ, Guan X, Bo H, Sun JY, Huang NW, He J, Zhang YK, et al: Andrographolide protects against LPS-induced acute lung injury by inactivation of NF- $\kappa \mathrm{B}$. PLoS One 8: e56407, 2013.

46. Ni S, Miao K, Zhou X, Xu N, Li C, Zhu R, Sun R and Wang Y: The involvement of follistatin-like protein 1 in osteoarthritis by elevating $\mathrm{NF}-\kappa \mathrm{B}$-mediated inflammatory cytokines and enhancing fibroblast like synoviocyte proliferation. Arthritis Res Ther 17: 91, 2015.

47. Lim CP and Cao X: Structure, function, and regulation of STAT proteins. Mol Biosyst 2: 536-550, 2006.

48. Lu R, Zhang YG and Sun J: STAT3 activation in infection and infection-associated cancer. Mol Cell Endocrinol 451: 80-87, 2017.

49. Ghilardi N, Ziegler S, Wiestner A, Stoffel R, Heim MH and Skoda RC: Defective STAT signaling by the leptin receptor in diabetic mice. Proc Natl Acad Sci USA 93: 6231-6235, 1996.
50. Devi SJ, Madhav MS, Kumar GR, Goel AK, Umakanth B, Jahnavi B and Viraktamath BC: Identification of abiotic stress miRNA transcription factor binding motifs (TFBMs) in rice. Gene 531: 15-22, 2013

51. Zamanighomi M, Lin Z, Wang Y, Jiang R and Wong WH: Predicting transcription factor binding motifs from DNA-binding domains, chromatin accessibility and gene expression data. Nucleic Acids Res 45: 5666-5677, 2017.

52. Eckert D, Buhl S, Weber S, Jäger R and Schorle H: The AP-2 family of transcription factors. Genome Biol 6: 246, 2005.

53. Xu X, Liu Z, Huang H, Zheng K, Hu X, Zhang Z and Qiu M: AP- $2 \alpha$ and AP- $2 \beta$ regulate dorsal interneuron specification in the spinal cord. Neuroscience 340: 232-242, 2017.

54. Suyama K, Kabuyama Y, Suzuki S, Kawasaki Y, Suzuki J, Suzuki $\mathrm{H}$ and Homma Y: Induction of transcription factor AP-2 by cytokines and prostaglandins in cultured mesangial cells. Am J Nephrol 21: 307-314, 2001.

55. Mi X, Tang W, Chen X, Liu F and Tang X: Mitofusin 2 attenuates the histone acetylation at collagen IV promoter in diabetic nephropathy. J Mol Endocrinol 57: 233-249, 2016.

56. Hobert O: Gene regulation by transcription factors and microRNAs. Science 319: 1785-1786, 2008.

57. He L and Hannon GJ: MicroRNAs: Small RNAs with a big role in gene regulation. Nat Rev Genet 5: 522-531, 2004.

58. Dieter C, Assmann TS, Costa AR, Canani LH, de Souza BM, Bauer AC and Crispim D: MiR-30e-5p and MiR-15a-5p expressions in plasma and urine of type 1 diabetic patients with diabetic kidney disease. Front Genet 10: 563, 2019.

59. Sun SQ, Zhang T, Ding D, Zhang WF, Wang XL, Sun Z, Hu LH, Qin SY, Shen LH and He B: Circulating MicroRNA-188, -30a, and $-30 \mathrm{e}$ as early biomarkers for contrast-induced acute kidney injury. J Am Heart Assoc 5: e004138, 2016.

60. Galicia JC, Naqvi AR, Ko CC, Nares $S$ and Khan AA: MiRNA-181a regulates Toll-like receptor agonist-induced inflammatory response in human fibroblasts. Genes Immun 15 333-337, 2014.

61. Yuan J, Ji H, Xiao F, Lin Z, Zhao X, Wang Z, Zhao J and Lu J: MicroRNA-340 inhibits the proliferation and invasion of hepatocellular carcinoma cells by targeting JAK1. Biochem Biophys Res Commun 483: 578-584, 2017.

62. Li Y, Fan X, He X, Sun H, Zou Z, Yuan H, Xu H, Wang C and Shi X: MicroRNA-4661 inhibits antiviral innate immune response by targeting interferon-alpha. Cell Mol Immunol 9: 497-502, 2012.

63. MaF, Liu X,LiD, Wang P, Li N, Lu L and Cao X: MicroRNA-4661 upregulates IL-10 expression in TLR-triggered macrophages by antagonizing RNA-binding protein tristetraprolin-mediated IL-10 mRNA degradation. J Immunol 184: 6053-6059, 2010.

64. Rout AK, Udgata SR, Dehury B, Pradhan SP, Swain HS, Behera BK and Das BK: Structural bioinformatics insights into the CARD-CARD interaction mediated by the mitochondrial antiviral-signaling protein of black carp. J Cell Biochem 120: 12534-12543, 2019.

65. Creagh EM and O'Neill LA: TLRs, NLRs and RLRs: A trinity of pathogen sensors that co-operate in innate immunity. Trends Immunol 27: 352-357, 2006.

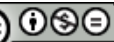

This work is licensed under a Creative Commons Attribution-NonCommercial-NoDerivatives 4.0 International (CC BY-NC-ND 4.0) License. 